\title{
Theoretical Basis of the Linear Nodal and Linear Characteristic Methods in the TORT Computer Code
}

\author{
R. L. Childs \\ W. A. Rhoades
}

DATE PUBLISHED_January 1993

Sponsored by

Defense Nuclear Agency

Prepared by the

OAK RIDGE NATIONAL LABORATORY

Oak Ridge, Tennessee 37831 managed by

MARTIN MARIETTA ENERGY SYSTEMS, INC.

for the

U.S. DEPARTMENT OF ENERGY under contract DE-AC05-84OR21400 


\section{CONTENTS}

LIST OF FIGURES . . . . . . . . . . . . . . . . . . . . . . v LIST OF TABLES . . . . . . . . . . . . . . . . . . . . . . . vii ACKNOWLEDGMENTS . . . . . . . . . . . . . . . ix ABSTRACT . . . . . . . . . . . . . . . . . . . xi

1. INTRODUCTION . . . . . . . . . . . . . . . . . . . . . . 1

2. DEVELOPMENT OF THE LINEAR NODAL EQUATIONS . . . . . 4

3. THE AUGMENTED WEIGHTED-DIFFERENCE FORM OF THE LINEAR NODAL METHOD . . . . . . . . . . . . . . . . 11

4. THE STEP LIMIT CORRECTION FOR LINEAR NODAL SOLUTIONS. 15

5. NEGATIVE FLUX PREVENTION . . . . . . . . . . . . . . 23

6. IMPLEMENTATION IN TORT . . . . . . . . . . . . . 30

7. THE LINEAR CHARACTERISTIC METHOD. . . . . . . . 32

8. TESTING AND APPLICATIONS . . . . . . . . . . . . . . . . 46

9. CONCLUDING COMMENTS . . . . . . . . . . . . . . . . 59

REFERENCES . . . . . . . . . . . . . . . . . . 61 


\section{LIST OF FIGURES}

$\underline{\text { Figure }}$

4.1. Comparison of Nodal and 0-Weighted TORT Results With Monte Carlo

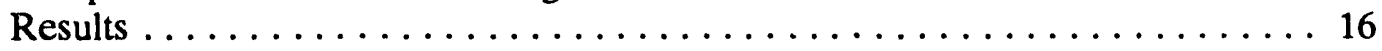

4.2. Comparison of Nodal and Weighted-Difference Results $\ldots \ldots \ldots \ldots \ldots \ldots$

4.3. Comparison of Nodal Results on Different Meshes $\ldots \ldots \ldots \ldots \ldots \ldots$

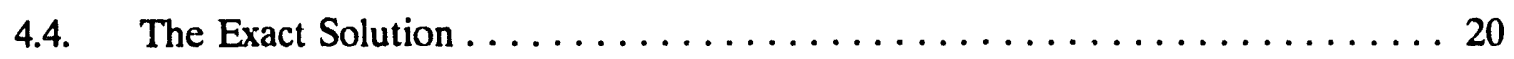

4.5. The Linear Nodal Solution With No Step Limit $\ldots \ldots \ldots \ldots \ldots \ldots$

4.6. The Linear Nodal Solution With the Step Limit $\ldots \ldots \ldots \ldots \ldots \ldots \ldots$

7.1. Two Dimensional Cases for the Characteristic Method ........... 34

7.2. Leakage Surface by Location on the $y=0$ Cell Face $\ldots \ldots \ldots \ldots \ldots$

7.3. Leakage Surface by location for Sources in $d y$ about $y \ldots \ldots \ldots \ldots \ldots \ldots$

8.1. Plan View of TVE Concrete Building $\ldots \ldots \ldots \ldots \ldots \ldots \ldots \ldots \ldots \ldots$

8.2. Flux In Zone Located In The Black, Center Above The Window Versus Mesh Size ................................ 49

8.3. Maximum Error for Different Methods and Mesh Sizes $\ldots \ldots \ldots \ldots \ldots \ldots$

8.4. Model of a Large Concrete Building. (Dimensions in $\mathrm{cm}) \ldots \ldots \ldots \ldots \ldots 2$

8.5. Cross Section of HFIR Vessel Showing Locations of Surveillance Capsules ... 56 


\section{LIST OF TABLES}

Table

8.1. Sodium-Steel-Concrete-Void Shielding Problem (Pointwise convergence criterion $\left.10^{-3}\right) \ldots \ldots \ldots \ldots \ldots \ldots \ldots \ldots \ldots \ldots \ldots \ldots \ldots \ldots \ldots \ldots$

8.2. Timing Results for Flux Procedures $\ldots \ldots \ldots \ldots \ldots \ldots \ldots \ldots \ldots \ldots$

8.3. Computing Speed for Flux Procedures $\ldots \ldots \ldots \ldots \ldots \ldots \ldots \ldots \ldots$

8.4. Calculated (C) and Experimental (E) Sample Activation in HFIR 


\section{ACKNOWLEDGMENTS}

The authors are grateful for the contributions of W. F. Walters, Los Alamos, who provided encouragement and pre-publication information to assist this effort, of Y. Y. Azmy and R. T. Santoro, Oak Ridge, for their remarks and review, and to S. P. Shriner and A. C. Alford, Oak Ridge, for their attention to the demanding preparation of the final manuscript. 


\begin{abstract}
Novel numerical procedures for solving the Boltzmann equation have been added to the Three Dimensional Oak Ridge Discrete Ordinates Transport Code (TORT). These procedures produce much more accuracy in the flux solutions for a given mesh size, or allow a smaller mesh to be used in order to reduce costs. The first method is a special adaptation of the linear nodal method proposed by Walters and O'Dell. The basic method has been extensively adapted in order to avoid numerical distortions that may occur in shielding problems. The second method is a characteristic procedure with linear expansion of sources and boundary flows. These methods are in widespread use in the TORT code.
\end{abstract}




\section{INTRODUCTION}

The TORT (1) computer code is a three-dimensional discrete ordinates neutron/photon transport code developed at Oak Ridge National Laboratory (ORNL). TORT is available from the Radiation Shielding Information Center and is now being used by a number of researchers at ORNL and elsewhere. An advanced feature of TORT is that linear nodal and linear characteristic methods of solving the discrete ordinates form of the transport equation are available in addition to the longer established weighted difference method. The purpose of the work described in this report is to provide a theoretical development of the linear nodal and linear characteristic methods found in TORT and also to study their accuracy and speed.

The authors became interested in the linear nodal method in 1981 after reading a paper by Walters and O'Dell of Los Alamos that compared the linear nodal, linear discontinuous, and diamond schemes for solving the transport equation in $(\mathrm{x}, \mathrm{y})$ geometry (2). This paper first introduced the linear nodal method which is a variation of a linear-linear nodal method (3) developed earlier by Walters and O'Dell. The linear nodal equations are obtained by dropping some of the terms in the linear-linear equations. Dropping these terms greatly simplifies the solution of the equations with little loss in accuracy. The motivation for using the linear nodal method in TORT was to obtain improved accuracy. Walters and O'Dell (2) estimated that linear nodal with an $\mathrm{N}$ by $\mathrm{N}$ mesh has roughly the same accuracy as diamond difference with a $4 \mathrm{~N}$ by $4 \mathrm{~N}$ mesh. Extending this estimate to three dimensions indicated that a diamond difference solution would require 64 times as many mesh cells as a linear nodal solution with the same accuracy.

In November of 1982, the authors met with Walters and O'Dell to discuss the prospects for using the linear nodal method in TORT. The meeting was very useful and led to the decision to pursue the linear nodal method further. One of Walters and O'Dell's recommendations was that we gain some experience with the method by working with two-dimensional $(x, y)$ geometry first. The method had not been extended to three-dirnensional $(x, y, z)$ geometry at this time. The single most valuable information obtained at the meeting was a listing of the FORTRAN used in a test version of TWOTRAN to implement the linear nodal method.

The linear nodal method was implemented in the DOT4 (4) computer code in 1983 (5). The most distinctive feature of this effort was our introduction of modifications to the linear nodal method that insured positive results. A test problem that included a void was also studied. Voids present a special challenge to the linear nodal method because special care must be taken to avoid division by zero. Near voids are also a problem because of roundoff errors. 
Work began on extending the linear nodal method to three-dimensional $(x, y, z)$ geometry for use in TORT in 1984 (6). This effort was aided when Walters provided a pre-publication copy of a paper that derived an augmented weighted diamond form of the linear nodal equations (7). Badruzzaman also developed an algorithm for three-dimensional nodal calculations (8) during this time period, but his solution could not be used for void or near void regions. Section 2 of this report contains a development of the linear nodal equations that follows very closely the derivation outlined by Walters (7). Section 3 of this report contains a derivation of the augmented weighted-difference solution of the linear nodal equations used in TORT. This derivation is strongly influenced by Walters' work, but the final form is slightly different.

When the linear nodal method was tested in TORT, very inaccurate results were noted for certain test problems. A modification to the definition of the linear nodal weighting coefficients was made that required the use of the step flux extrapolation model for certain unusual conditions. This "step limit" correction is described in Section 4. The step limit correction is unique to TORT. The linear nodal method would not be suitable for use in a general purpose code without this extension.

The negative flux prevention procedures used in TORT are described in Section 5. The requirement of non-negative flux solutions is considered essential in the judgment of the authors. The procedures used in TORT are based upon our finding that the linear nodal method always gives non-negative results for a mesh cell when the incoming flux distributions on the cell boundaries and the source distribution within the cell are flat and non-negative. When the linear expansion coefficients for these fluxes and sources are large enough to cause the average fluxes in the cell or on the cell boundaries to be negative, the coefficients are reduced a sufficient amount to prevent the negative results.

Section 6 describes the numerical approximations used to implement the linear nodal method in TORT and also the way anisotropic scattering is treated. The derivations in sections 2 and 3 find two functions defined using exponentials that must be evaluated in linear nodal calculations. Numerical approximations for these functions that are accurate for void, near-void, and high cross section mesh cells are used in TORT. Anisotropic scattering is treated in a simple way that assumes the spatial distribution and the angular distribution in a mesh cell are separable. This treatment for anisotropic scattering is believed to reduce accuracy in a k-calculation test case.

After the linear nodal method was installed in TORT, the possibility of using analytic (characteristic) solutions of the transport equation, rather than nodal approximations, was studied (9). A review of characteristic methods for solving the 
transport equation is found in Reference 10. Larsen and Alcouffe have studied a linear characteric method in $(\mathrm{x}, \mathrm{y})$ geometry $(11)$. The derivation of the linear characteristic equations used in TORT are found in Section 7. The derivation of the characteristic method contains fewer approximations than the linear nodal derivation. Non-physical results such as the step limit problem with the linear nodal method are unlikely with the characteristic method. Negative flux prevention is also more straightforward. The characteristic method in TORT is a hybrid method because characteristic solutions are used to obtain average values for fluxes in the mesh cell and on the cell boundaries and nodal approximations are used to obtain the linear expansion coefficients for these fluxes.

Section 8 discusses the results of practical testing and applications. 


\section{DEVELOPMENT OF THE LINEAR NODAL EQUATIONS}

Consider the three-dimensional cartesian space cell (node) described by

$$
\begin{aligned}
& x_{L} \leq x \leq x_{R} \\
& y_{\text {in }} \leq y \leq y_{\text {out }} \\
& z_{B} \leq z \leq z_{T}
\end{aligned}
$$

with

$$
\begin{aligned}
& \Delta x=x_{R}-x_{L} \\
& \Delta y=y_{\mathrm{out}}-y_{\mathrm{in}} \\
& \Delta z=z_{T}-z_{B}
\end{aligned}
$$

and

$$
\begin{aligned}
& \bar{x}=\frac{x_{L}+x_{R}}{2} \\
& \bar{y}=\frac{y_{\text {in }}+y_{\text {out }}}{2} \\
& \bar{z}=\frac{z_{B}+z_{T}}{2} .
\end{aligned}
$$

The subscripts used here are $\mathrm{L}=$ left, $\mathrm{R}=$ right, $\mathrm{B}=$ bottom and $\mathrm{T}=$ top. The discrete ordinates form of the transport equation for a single direction is

$$
\mu \frac{\partial \psi(x, y, z)}{\partial x}+\xi \frac{\partial \psi(x, y, z)}{\partial y}+\eta \frac{\partial \psi(x, y, z)}{\partial z}+\sigma \psi(x, y, z)=S(x, y, z)
$$

Here $\psi(x, y, z)$ is the angular flux at point $(x, y, z)$. Subscripts are used to indicate fluxes on the boundaries of the space cell. Thus,

$$
\begin{aligned}
\psi_{L}(y, z) & =\psi\left(x_{L}, y, z\right), \\
\psi_{R}(y, z) & =\psi\left(x_{R}, y, z\right), \\
\psi_{\text {in }}(x, z) & =\psi\left(x, y_{\text {in }}, z\right), \\
\psi_{\text {out }}(x, z) & =\psi\left(x, y_{\text {out }}, z\right) \\
\psi_{B}(x, y) & =\psi\left(x, y, z_{B}\right), \\
\text { and } \quad \psi_{T}(x, y) & =\psi\left(x, y, z_{T}\right) .
\end{aligned}
$$


The dropping of one or more of the dependent variables indicates an average over that variable. Examples are

$$
\begin{aligned}
\psi(x, y) & =\frac{1}{\Delta z} \int_{z_{B}}^{z_{T}} \psi(x, y, z) d z \\
\psi(x) & =\frac{1}{\Delta y} \int_{y_{\text {in }}}^{y_{\text {out }}} \psi(x, y) d y \\
\psi_{a v} & =\frac{1}{\Delta x} \int_{x_{L}}^{x_{R}} \psi(x) d x \\
\psi_{L}(y) & =\frac{1}{\Delta z} \int_{z_{B}}^{z_{T}} \psi_{L}(y, z) d z
\end{aligned}
$$

and

$$
\psi_{L}=\frac{1}{\Delta y} \int_{y_{\text {in }}}^{y_{\text {out }}} \psi_{L}(y) d y
$$

The first $x, y$, or $z$ moment is indicated by the appropriate superscript. Examples are

$$
\begin{aligned}
\psi^{x}(y, z) & \equiv \frac{6}{\Delta x^{2}} \int_{x_{L}}^{x_{R}} \psi(x, y, z)(x-\bar{x}) d x \\
\psi_{B}^{x}(y) & \equiv \frac{6}{\Delta x^{2}} \int_{x_{L}}^{x_{R}} \psi_{B}(x, y)(x-\bar{x}) d x \\
\psi^{z}(x, y) & \equiv \frac{6}{\Delta z^{2}} \int_{z_{B}}^{z_{T}} \psi(x, y, z)(z-\bar{z}) d z \\
\text { and } \psi_{R}^{z}(y) & \equiv \frac{6}{\Delta z^{2}} \int_{z_{B}}^{z_{T}} \psi_{R}(y, z)(z-\bar{z}) d z
\end{aligned}
$$

When first moment quantities are averaged over a variable, the variable is dropped as before. Examples are

$$
\begin{aligned}
\psi^{x}(y) & =\frac{1}{\Delta z} \int_{z_{B}}^{z_{T}} \psi^{x}(y, z) d z \\
\psi^{x} & =\frac{1}{\Delta y} \int_{y_{\mathrm{in}}}^{y_{\mathrm{out}}} \psi^{x}(y) d y
\end{aligned}
$$

and

$$
\psi_{R}^{z}=\frac{1}{\Delta y} \int_{y_{\text {in }}}^{y_{\text {out }}} \psi_{R}^{z}(y) d y .
$$


The particle balance equation is obtained by applying the operator $\frac{1}{\Delta x \Delta y \Delta z} \int_{x_{L}}^{x_{R}} \int_{y_{\text {in }}}^{y_{\text {out }}} \int_{z_{B}}^{z_{T}} d x d y d z$ to Equation (2.1). The result is

$$
\sigma \psi_{a v}=S_{a v}+A_{x}\left(\psi_{L}-\psi_{R}\right)+A_{y}\left(\psi_{\text {in }}-\psi_{\text {out }}\right)+A_{z}\left(\psi_{B}-\psi_{T}\right),
$$

$$
\text { where } \begin{aligned}
A_{x} & =\frac{\mu}{\Delta x}, \\
A_{y} & =\frac{\xi}{\Delta y}, \\
A & =\frac{\eta}{\Delta z} .
\end{aligned}
$$

An exact differential equation for $\psi(x)$ is obtained by applying the operator $\frac{1}{\Delta y \Delta z} \int_{y_{\text {in }}}^{y_{\text {out }}} \int_{z_{B}}^{z_{T}} d y d z$ to Equation (2.1). This result is

$$
\begin{aligned}
\mu \frac{d \psi(x)}{d x}+\sigma \psi(x)=S(x) & +A_{y}\left[\psi_{\text {in }}(x)-\psi_{\text {out }}(x)\right] \\
& +A_{z}\left[\psi_{B}(x)-\psi_{T}(x)\right] .
\end{aligned}
$$

There are analogous equations for $\psi(y)$ and $\psi(z)$.

An equation describing the first $\mathrm{x}$-moment is obtained by applying the operator $\frac{6}{\Delta x^{2}} \int_{x_{L}}^{x_{R}}(x-\bar{x}) d x$ to Equation (2.1). This yields

$$
\begin{aligned}
& \xi \frac{\partial \psi^{x}(y, z)}{\partial y}+\eta \frac{\partial \psi^{x}(y, z)}{\partial z}+\sigma \psi^{x}(y, z)= \\
& S^{x}(y, z)+3 A_{x}\left[2 \psi(y, z)-\psi_{R}(y, z)-\psi_{L}(y, z)\right]
\end{aligned}
$$

The $\mathrm{x}$-moment balance equation is obtained by applying the operator $\frac{1}{\Delta y \Delta z} \int_{y_{\text {in }}}^{y_{\text {out }}} \int_{z_{B}}^{z_{T}} d y d z$ to Equation (2.4). This yields

$$
\begin{aligned}
\sigma \psi^{x}=S^{x} & +A_{y}\left(\psi_{\mathrm{in}}^{x}-\psi_{\text {out }}^{x}\right) \\
& +A_{z}\left(\psi_{B}^{x}-\psi_{T}^{x}\right) \\
& +3 A_{x}\left(2 \psi_{a v}-\psi_{R}-\psi_{L}\right) .
\end{aligned}
$$

The $y$-moment and $z$-moment balance equations are 


$$
\begin{aligned}
\sigma \psi^{y}=S^{y} & +A_{x}\left(\psi_{L}^{y}-\psi_{R}^{y}\right) \\
& +A_{z}\left(\psi_{B}^{y}-\psi_{T}^{y}\right) \\
& +3 A_{y}\left(2 \psi_{a v}-\psi_{\text {out }}-\psi_{\text {in }}\right)
\end{aligned}
$$

and

$$
\begin{aligned}
\sigma \psi^{z}=S^{z} & +A_{x}\left(\psi_{L}^{z}-\psi_{R}^{z}\right) \\
& +A_{y}\left(\psi_{\mathrm{in}}^{z}-\psi_{\text {out }}^{z}\right) \\
& +3 A_{z}\left(2 \psi_{a v}-\psi_{T}-\psi_{B}\right)
\end{aligned}
$$

An exact differential equation for $\psi^{x}(z)$ is obtained by applying the operator $\frac{1}{\Delta y} \int_{y_{\text {in }}}^{y_{\text {out }}} d y$ to Equation (2.4). This is

$$
\begin{aligned}
\eta \frac{d \psi^{x}(z)}{d z}+\sigma \psi^{x}(z)=S^{x}(z) & +A_{y}\left[\psi_{\text {in }}^{x}(z)-\psi_{\text {out }}^{x}(z)\right] \\
& +3 A_{x}\left[2 \psi(z)-\psi_{R}(z)-\psi_{L}(z)\right]
\end{aligned}
$$

There are five equations analogous to Equation (2.6) for $\psi^{x}(y), \psi^{y}(x), \psi^{y}(z), \psi^{z}(x)$, and $\psi^{z}(y)$.

The four balance equations (Equation (2.2) and Equations (2.5)) contain 13 known quantities and 13 unknown quantities for each node. In a local coordinate system in which $\mu, \xi$, and $\eta$ are positive, the known quantities are

$$
\begin{aligned}
& S, S^{x}, S^{y}, S^{z}, \\
& \psi_{L}, \psi_{L}^{y}, \psi_{L}^{z}, \\
& \psi_{\mathrm{in}}, \psi_{\mathrm{in}}^{x}, \psi_{\mathrm{in}}^{z}, \\
\text { and } & \psi_{B}, \psi_{B}^{x}, \psi_{B}^{y} .
\end{aligned}
$$

The unknown quantities are

$$
\begin{aligned}
& \psi_{a v}, \psi^{x}, \psi^{y}, \psi^{z}, \\
& \psi_{R}, \psi_{R}^{y}, \psi_{R}^{z}, \\
& \psi_{\text {out }}, \psi_{\text {out }}^{x}, \psi_{\text {out }}^{z}, \\
& \text { and } \psi_{T}, \psi_{T}^{x}, \psi_{T}^{y}
\end{aligned}
$$


In addition to the four balance equations, nine additional equations are needed to solve for the unknowns. The next three equations are obtained from Equation (2.3) and its $y$ and $z$ counterparts by substituting linear expansions. The linear expansions for the five functions of $x$ on the right hand side of Equation (2.3) are

$$
\begin{aligned}
S(x) & =S_{a v}+S^{x} \frac{2(x-\bar{x})}{\Delta x}, \\
\psi_{\text {in }}(x) & =\psi_{\text {in }}+\psi_{\text {in }}^{x} \frac{2(x-\bar{x})}{\Delta x}, \\
\psi_{\text {out }}(x) & =\psi_{\text {out }}+\psi_{\text {out }}^{x} \frac{2(x-\bar{x})}{\Delta x}, \\
\psi_{B}(x) & =\psi_{B}+\psi_{B}^{x} \frac{2(x-\bar{x})}{\Delta x}, \\
\text { and } \psi_{T}(x) & =\psi_{T}+\psi_{T}^{x} \frac{2(x-\bar{x})}{\Delta x} .
\end{aligned}
$$

Substituting Equations (2.7) into Equation (2.3) yields

$$
\begin{aligned}
\mu \frac{d \psi(x)}{d x} & +\sigma \psi(x)=\left[S_{a v}+A_{y}\left(\psi_{\text {in }}-\psi_{\text {out }}\right)+A_{z}\left(\psi_{B}-\psi_{T}\right)\right] \\
& +\frac{2(x-\bar{x})}{\Delta x}\left[S^{x}+A_{y}\left(\psi_{\text {in }}^{x}-\psi_{\text {out }}^{x}\right)+A_{z}\left(\psi_{B}^{x}-\psi_{T}^{x}\right)\right]
\end{aligned}
$$

Equation (2.8) can be put into a form that contains no reference to the $y$ and $z$ boundaries using Equation (2.2) (the balance equation) and Equation (2.5a) (the $\mathrm{x}$-moment balance equation):

$$
\begin{aligned}
\mu \frac{d \psi(x)}{d x}+\sigma \psi(x) & =\left[\sigma \psi_{a v}+A_{x}\left(\psi_{R}-\psi_{L}\right)\right] \\
& +\frac{2(x-\bar{x})}{\Delta x}\left[\sigma \psi^{x}+3 A_{x}\left(\psi_{R}+\psi_{L}-2 \psi_{a v}\right)\right]
\end{aligned}
$$

The solution of this ordinary differential equation evaluated at $x_{R}$ is

$$
\begin{aligned}
\psi_{R}=\psi_{L} e^{-\epsilon_{x}} & +P 0\left(\epsilon_{x}\right)\left(\epsilon_{x} \psi_{a v}+\psi_{R}-\psi_{L}\right) \\
& +\left[2 P 1\left(\epsilon_{x}\right)-P 0\left(\epsilon_{x}\right)\right]\left[\epsilon_{x} \psi^{x}+3\left(\psi_{R}+\psi_{L}-2 \psi_{a v}\right)\right]
\end{aligned}
$$


where

$$
\begin{aligned}
\epsilon_{x}=\frac{\sigma}{A_{x}} & =\frac{\sigma \Delta x}{\mu} \\
P 0\left(\epsilon_{x}\right) & =\frac{1-e^{-\epsilon_{x}}}{\epsilon_{x}} \\
\text { and } P 1\left(\epsilon_{x}\right) & =\frac{1-P 0\left(\epsilon_{x}\right)}{\epsilon_{x}} .
\end{aligned}
$$

There are analogou: equations for $\psi_{\text {out }}$ and $\psi_{T}$.

The final six equations are obtained from Equation (2.6) and its five analogs. The linear nodal result is obtained by substituting average values for the functions of $z$ on the right hand side of Equation (2.6). (A more complicated set of equations called the linear-linear method is obtained if linear expansions instead of average values are substituted.) With the average values substituted, Equation (2.6) becomes

$$
\eta \frac{d \psi^{x}(z)}{d z}+\sigma \psi^{x}(z)=S^{x}+A_{y}\left(\psi_{\mathrm{in}}^{x}-\psi_{\mathrm{out}}^{x}\right)+3 A_{x}\left(2 \psi_{a v}-\psi_{R}-\psi_{L}\right)
$$

The $\mathrm{x}$-moment balance equation (Equation 2.5a) is used to put Equation (2.11) in a form that contains no reference to the $\mathrm{x}$ and $\mathrm{y}$ boundaries:

$$
\eta \frac{d \psi^{x}(z)}{d z}+\sigma \psi^{x}(z)=\sigma \psi^{x}+A_{z}\left(\psi_{T}^{x}-\psi_{B}^{x}\right)
$$

The solution of this differential equation evaluated at $z_{T}$ is

$$
\psi_{T}^{x}=\psi_{B}^{x} e^{-\epsilon_{z}}+P 0\left(\epsilon_{z}\right)\left(\epsilon_{z} \psi^{x}+\psi_{T}^{x}-\psi_{B}^{x}\right)
$$

where $\epsilon_{z}=\frac{\sigma}{A_{z}}$.

Some simple algebra results in

$$
\psi_{T}^{x}=\psi_{B}^{x}+F\left(\epsilon_{z}\right)\left(\psi^{x}-\psi_{B}^{x}\right),
$$

where $F\left(\epsilon_{z}\right)=\frac{P 0\left(\epsilon_{z}\right)}{P 1\left(\epsilon_{z}\right)}$. 
The analogous relationship for $\psi_{\text {out }}^{x}$ is

$$
\psi_{\mathrm{out}}^{x}=\psi_{\mathrm{in}}^{x}+F\left(\epsilon_{y}\right)\left(\psi^{x}-\psi_{\mathrm{in}}^{x}\right)
$$

There are also similar equations for $\psi_{R}^{y}, \psi_{T}^{y}, \psi_{R}^{z}$, and $\psi_{\text {out }}^{z}$.

In the limit of small $\epsilon_{z}$, Equation (2.14a) becomes the diamond relationship

$$
\psi^{x}=\frac{\psi_{B}^{x}+\psi_{T}^{x}}{2}
$$

In the limit of large $\epsilon_{z}$, Equation (2.14a) becomes the step relationship

$$
\psi_{T}^{x}=\psi^{x}
$$




\section{THE AUGMENTED WEIGHTED-DIFFERENCE FORM OF THE LINEAR NODAL METHOD}

Equation (2.10) contains three unknown quantities, $\psi_{R}, \psi_{a v}$, and $\psi^{*}$. In order to obtain the desired weighted difference form, an expression for $\psi^{x}$ containing only two unknowns, $\psi_{a v}$ and $\psi_{R}$, is needed. This result is obtained by substituting Equations (2.14) into the $\mathrm{x}$-moment balance equation (Equation (2.5a)) to obtain:

$$
\sigma \psi^{x}=S^{x}+\rho_{y}\left(\psi_{\mathrm{in}}^{x}-\psi^{x}\right)+\rho_{z}\left(\psi_{B}^{x}-\psi^{x}\right)+3 A_{x}\left(2 \psi_{a v}-\psi_{R}-\psi_{L}\right),
$$

where

$$
\begin{aligned}
& o_{y}=A_{y} F\left(\epsilon_{y}\right) \text { and } \\
& \rho_{z}=A_{z} F\left(\epsilon_{z}\right) .
\end{aligned}
$$

This equation can be solved for $\psi^{x}$ to obtain

$$
\psi^{x}=\frac{3\left(2 \psi_{a v}-\psi_{R}-\psi_{L}\right)+Q^{x} / A_{x}}{D_{x}},
$$

where

$$
Q^{x}=S^{x}+\rho_{y} \psi_{\mathrm{in}}^{x}+\rho_{z} \psi_{B}^{x}
$$

and

$$
D_{x}=\frac{\sigma+\rho_{y}+\rho_{z}}{A_{x}}=\epsilon_{x}+\frac{\rho_{y}+\rho_{z}}{A_{x}}
$$

Substituting Equation (3.2) into Equation (2.10) and grouping terms results in 


$$
\begin{aligned}
& \psi_{R}\left[\left(1-P 0\left(\epsilon_{x}\right)\right)-3\left(2 P 1\left(\epsilon_{x}\right)-P 0\left(\epsilon_{x}\right)\right)\left(1-\frac{\epsilon_{x}}{D_{x}}\right)\right]= \\
& \psi_{L}\left[e^{-\epsilon_{x}}-P 0\left(\epsilon_{x}\right)+3\left(2 P 1\left(\epsilon_{x}\right)-P 0\left(\epsilon_{x}\right)\right)\left(1-\frac{\epsilon_{x}}{D_{x}}\right)\right] \\
& +\psi_{a v}\left[\epsilon_{x} P 0\left(\epsilon_{x}\right)-6\left(2 P 1\left(\epsilon_{x}\right)-P 0\left(\epsilon_{x}\right)\right)\left(1-\frac{\epsilon_{x}}{D_{x}}\right)\right] \\
& +Q^{x}\left[\left(2 P 1\left(\epsilon_{x}\right)-P 0\left(\epsilon_{x}\right)\right) \frac{\epsilon_{x}}{A_{x} D_{x}}\right]
\end{aligned}
$$

A modest amount of algebra is required to show that Equation (3.4) can be cast in the following weighted difference form:

$$
\psi_{R}=\left(2-\alpha_{x} D_{x}\right) \psi_{a v}+\left(\alpha_{x} D_{x}-1\right) \psi_{L}+\frac{\alpha_{x} Q^{x}}{A_{x}}
$$

where

$$
\alpha_{x}=\frac{\frac{\epsilon_{x}}{D_{x}}\left(2 P 1\left(\epsilon_{x}\right)-P 0\left(\epsilon_{x}\right)\right)}{\left(1-P 0\left(\epsilon_{x}\right)\right)-3\left(2 P 1\left(\epsilon_{x}\right)-P 0\left(\epsilon_{x}\right)\right)\left(1-\frac{\epsilon_{x}}{D_{x}}\right)}
$$

The result for $\alpha_{x}$ can be simplified to

$$
\alpha_{x}=\frac{1}{3+G\left(\epsilon_{x}\right) D_{x}}
$$

$$
\text { where } \begin{aligned}
\quad G\left(\epsilon_{x}\right) & =\frac{P 2\left(\epsilon_{x}\right)-P 3\left(\epsilon_{x}\right)}{P 1\left(\epsilon_{x}\right)-P 2\left(\epsilon_{x}\right)} \\
P 2\left(\epsilon_{x}\right) & =\frac{1-2 P 1\left(\epsilon_{x}\right)}{\epsilon_{x}} \\
\text { and } \quad P 3\left(\epsilon_{x}\right) & =\frac{1-3 P 2\left(\epsilon_{x}\right)}{\epsilon_{x}}
\end{aligned}
$$

Equation (3.6) is valid for small $\epsilon_{x}$ and also for $\epsilon_{x}=0$, an important consideration for problems containing a vacuum.

Note that the last term in Equation (3.5) involves the $x$-moment of the source and the incoming boundary fluxes. If this term is dropped, a true weighted difference 
form remains. As will be discussed later, this weighted difference form will always give positive results for $\tau_{R}, \psi_{\text {out }}, \psi_{T}$, and $\psi_{\text {av }}$ given positive values of $\psi_{L}, \psi_{\text {in }}, \psi_{R}$, and $\mathrm{S}$. Also note that $\alpha_{x} D_{x}$ is independent of the boundary flux and source values. However, dropping the $Q^{x}$ term results in a method that is less accurate than conventional weighted difference because a flat source assumption allows too much transmission in shielding problems.

A more convenient form of Equation (3.5) is

$$
\psi_{R}=\gamma_{x} \psi_{a v}+\left(1-\gamma_{x}\right) \psi_{L}+\frac{\alpha_{x} Q^{x}}{A_{x}}
$$

where

$$
\gamma_{x}=2-\alpha_{x} D_{x}
$$

analogous relationships for $\psi_{\text {out }}$ and $\psi_{T}$ are

$$
\psi_{\text {out }}=\gamma_{y} \psi_{a v}+\left(1-\gamma_{y}\right) \psi_{\mathrm{in}}+\frac{\alpha_{y} Q^{y}}{A_{y}}
$$

and

$$
\psi_{T}=\gamma_{z} \psi_{a v}+\left(1-\gamma_{z}\right) \psi_{B}+\frac{\alpha_{z} Q^{z}}{A_{z}}
$$

All of the terms for the $y$ and $z$ direction are defined in the same way that the $x$ direction terms are defined. For example,

$$
Q^{y}=S^{y}+\rho_{x} \psi_{L}^{y}+\rho_{z} \psi_{B}^{y}
$$

and

$$
Q^{z}=S^{z}+\rho_{x} \psi_{L}^{z}+\rho_{y} \psi_{\mathrm{in}}^{z}
$$

Equations (3.7) can be substituted into the balance equation (Equation 2.2) to obtain an expression for $\psi_{a v}$. This result is

$$
\psi_{a v}=\frac{S_{a v}+\beta_{x} \psi_{L}+\beta_{y} \psi_{\mathrm{in}}+\beta_{z} \psi_{B}-\alpha_{x} Q^{x}-\alpha_{y} Q^{y}-\alpha_{z} Q^{z}}{D}
$$




$$
\text { where } \quad \begin{aligned}
\beta_{x} & =A_{x} \gamma_{x}, \\
\beta_{y} & =A_{y} \gamma_{y} \\
\beta_{z} & =A_{z} \gamma_{z} \\
\text { and } \quad D & =\sigma+\beta_{x}+\beta_{y}+\beta_{z} .
\end{aligned}
$$

Once $\psi_{a v}$ is known, $\psi_{R}, \psi_{\text {out }}$, and $\psi_{T}$ can be calculated from Equations (3.7). Once these are known, $\psi^{x}, \psi^{y}$, and $\psi^{z}$ can be calculated using Equation (3.2) and its $y$ and $z$ analogs. Finally, $\psi_{R}^{y}, \psi_{R}^{z}, \psi_{\text {out }}^{x}, \psi_{\text {out }}^{z}, \psi_{T}^{x}$ and $\psi_{T}^{z}$ are calculated from Equations(2.14) and their analogs. At this point, all of the thirteen unknowns have been obtained for one discrete angle and one mesh cell. 


\section{THE STEP LIMIT CORRECTION FOR LINEAR NODAL SOLUTIONS}

The initial implementation of the linear nodal method in the TORT code omitted the $Q^{x}, Q^{y}$, and $Q^{z}$ terms in Equations (3.7) and (3.9). This simplification allowed the developers to obtain numerical results very quickly with minimal programming. Even these preliminary results were very encouraging as shown in Figure 4.1. However, additional results revealed a very serious problem. This is shown in Figure 4.2. Note the thin interval at position 0.0 where the weighted difference result drops and the nodal result has a non-physical peak. Figure 4.3 shows the nodal result from Figure 4.2 along with a coarse mesh result. Here the nodal result has a false peak which is a factor of two too high. After the entire linear nodal method was implemented in TORT this problem still remained. The interval where the peak occurs contains a thin partition, and there is no physical reason for the peak.

The reason for this problem was not immediately apparent, but the values of $\gamma_{x}, \gamma_{y}$, and $\gamma_{z}$ were immediately suspect. Note in Equation (3.7a) with $Q^{x}=0$ that a value of two for $\gamma_{x}$ results in the diamond relationship

$$
\psi_{a v}=\frac{\psi_{R}+\psi_{L}}{2}
$$

while a value of one for $\gamma_{x}$ results in the step relationship

$$
\psi_{R}=\psi_{a v}
$$

The equation for $\gamma_{x}$ is

$$
\gamma_{x}=2-\alpha_{x} D_{x}
$$

Substituting the result for $\alpha_{x}$ from Equation (3.6) yields

$$
\gamma_{x}=2-\frac{D_{x}}{3+G\left(\epsilon_{x}\right) D_{x}}
$$




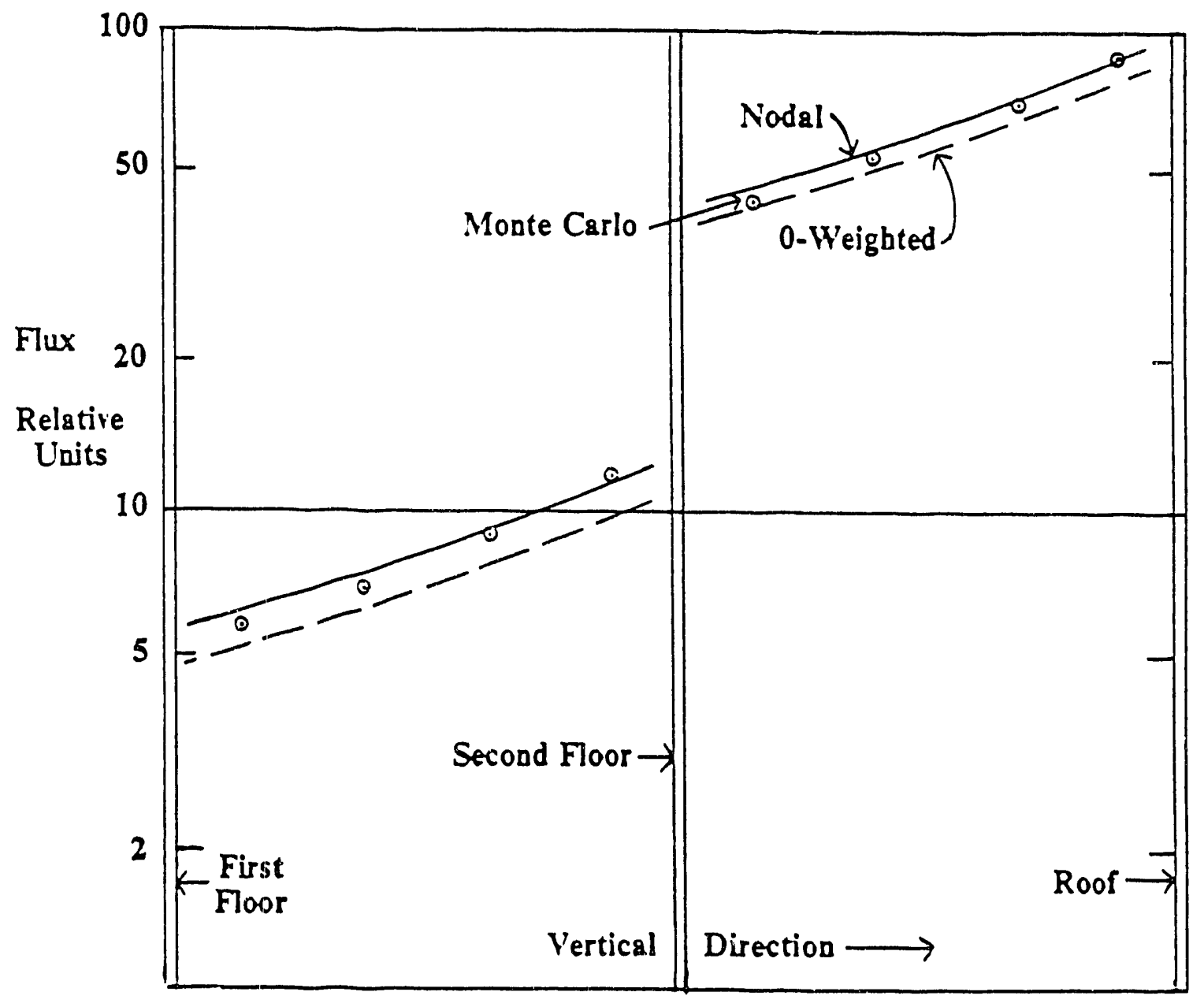

Figure 4.1 Comparison of Nodal and 0-Weighted TORT Results With Monte Carlo Results. 


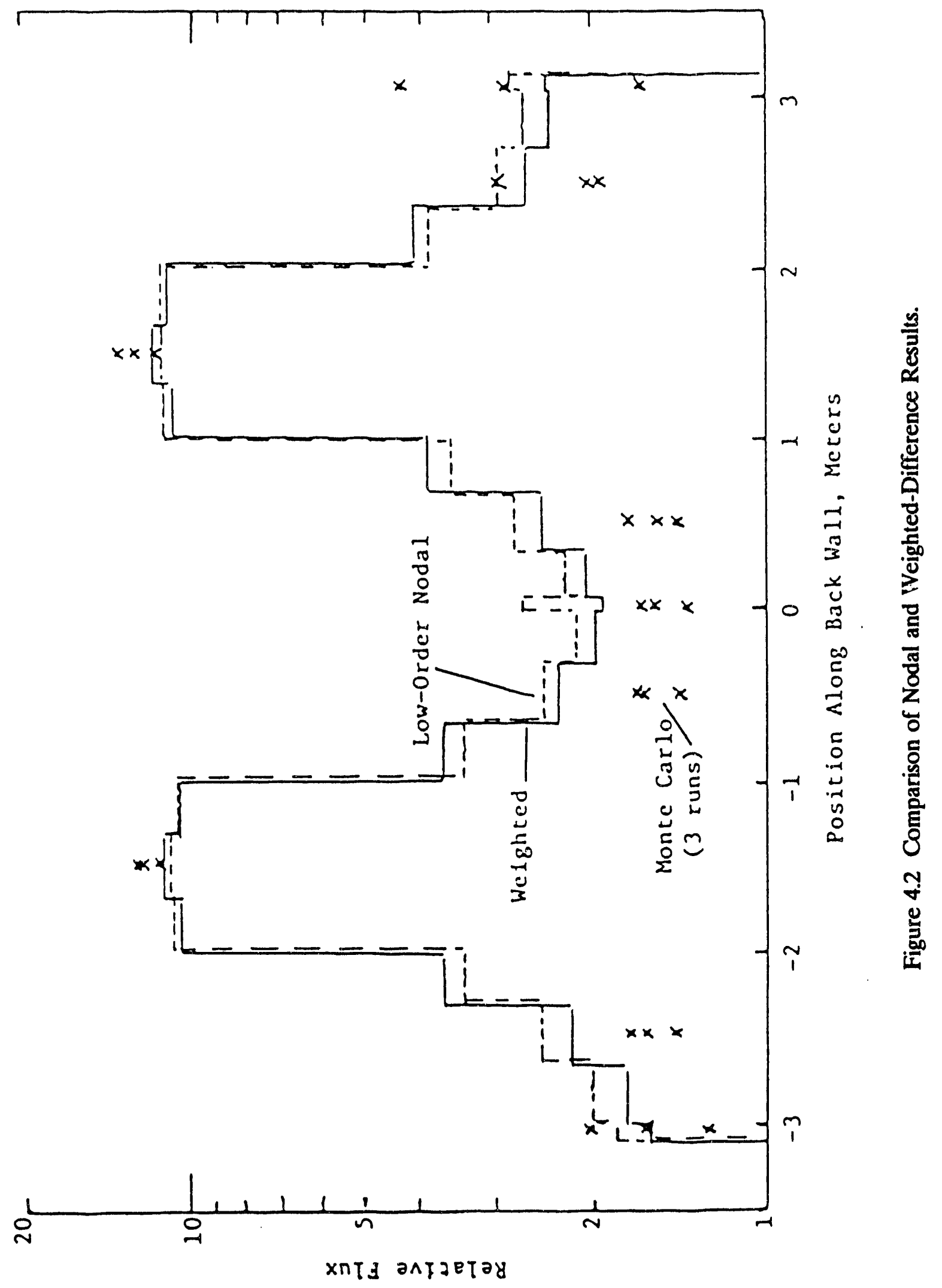




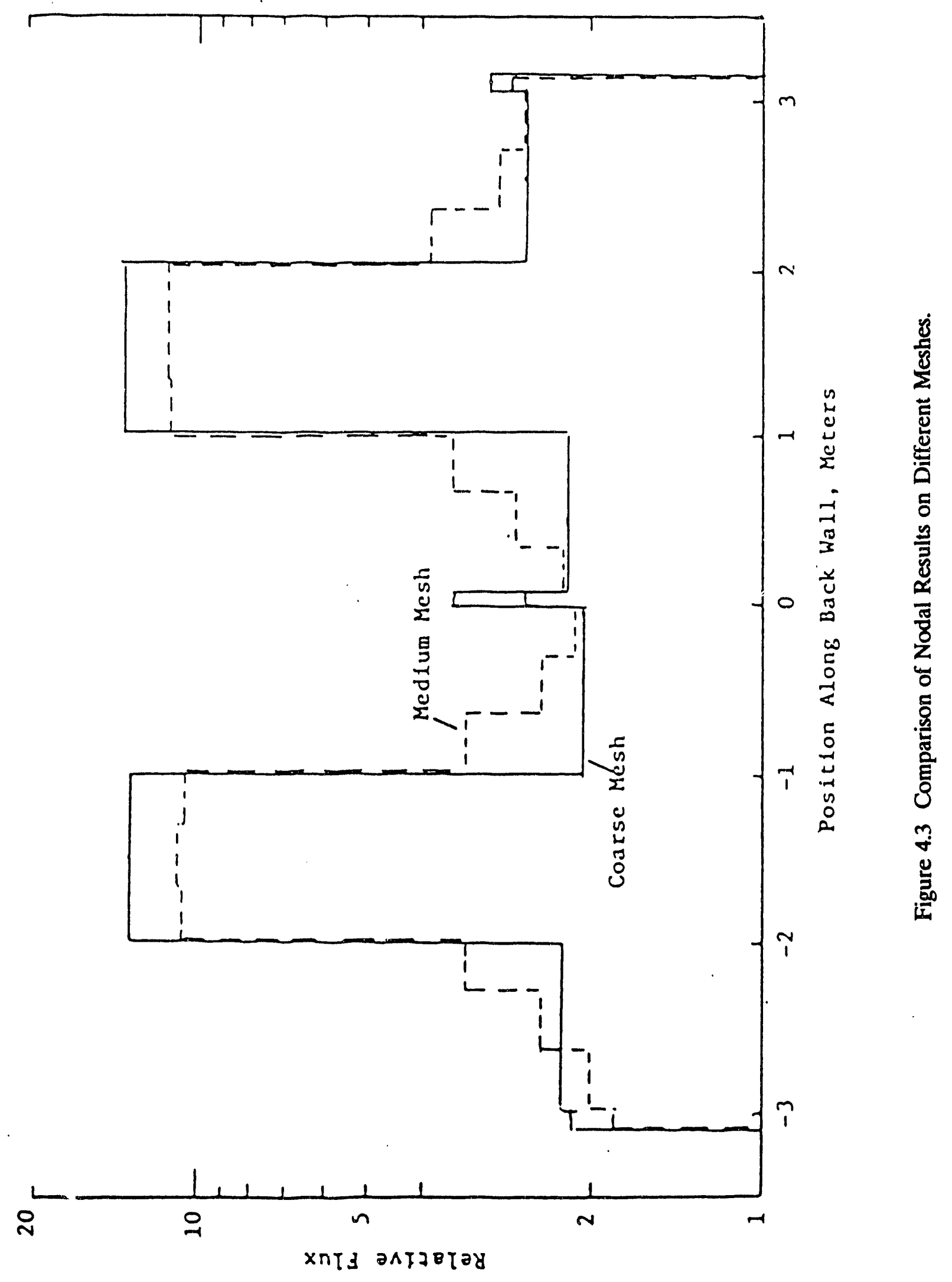


The function $G\left(\epsilon_{x}\right)$ has a minimum value of $\frac{1}{2}$ when $\epsilon_{x}$ is zero, and $D_{x}$ can be large at times. Thus, $\gamma_{x}$ can approach zero. $\gamma_{x}$ approaching zero gives the result

$$
\psi_{R}=\psi_{L}
$$

which is shown to be incorrect later in this section. In order to impose the step limit, the definition of $\alpha_{x}$ was modified as follows

$$
\alpha_{x}=\min \left(\frac{1}{D_{x}}, \frac{1}{3+G\left(\epsilon_{x}\right) D_{x}}\right) .
$$

This places an upper limit on $\alpha_{x}$ such that

$$
\begin{aligned}
\alpha_{x} D_{x} & \leq 1 \text { and } \\
\gamma_{x} & \geq 1 .
\end{aligned}
$$

The step limit correction was first tested in the calculation shown in Figures 4.2 and 4.3 and the non-physical peak disappeared.

The effect of the step limit correction is clearly demonstrated by considering a two-dimensional case in which a square mesh cell containing a void has a flat source on the left boundary. Figure 4.4 shows the exact solution for this problem as a function of the angle between the discrete direction and the normal to the left boundary. When this angle is zero, all particles entering the left boundary exit through the right boundary and $\psi_{R}$ equals $\psi_{L}$. When the angle equals 45 degrees, all of the particles exit through the top boundary and $\psi_{R}$ is zero.

Figure 4.5 shows the linear nodal result for this problem without the step limit correction along with the correct solution. The linear nodal result is completely non-physical for angles near 90 degrees. Figures 4.6 shows the linear nodal result with the step limit correction. While this result still has non-zero values for angles greater than 45 degrees, it does approach the correct limit near 90 degrees, and the accuracy near 0 degrees is also improved. 


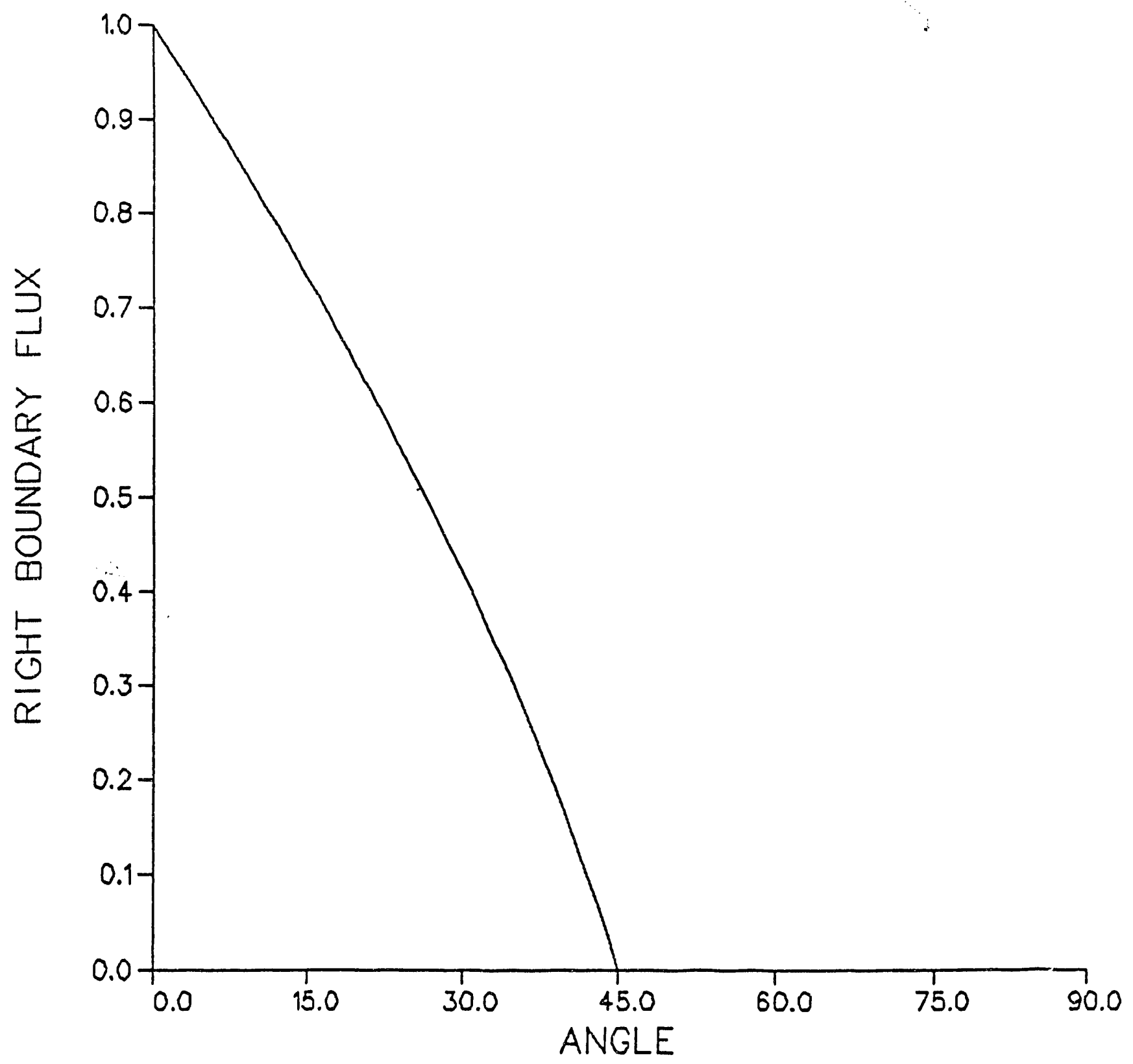

Figure 4.4 The Exact Solution 


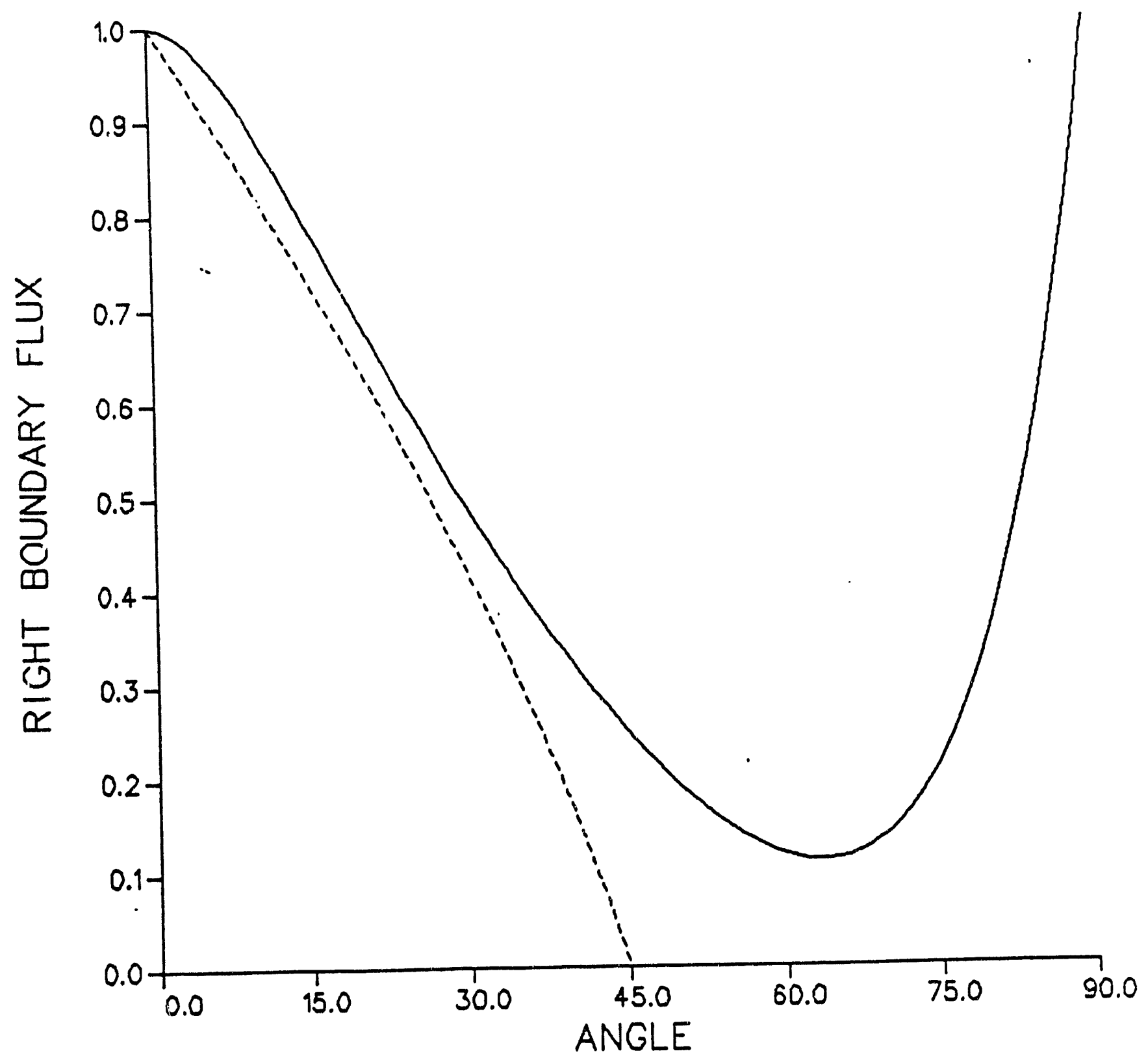

Figure 4.5 The Linear Nodal Solution With No Step Limit. 


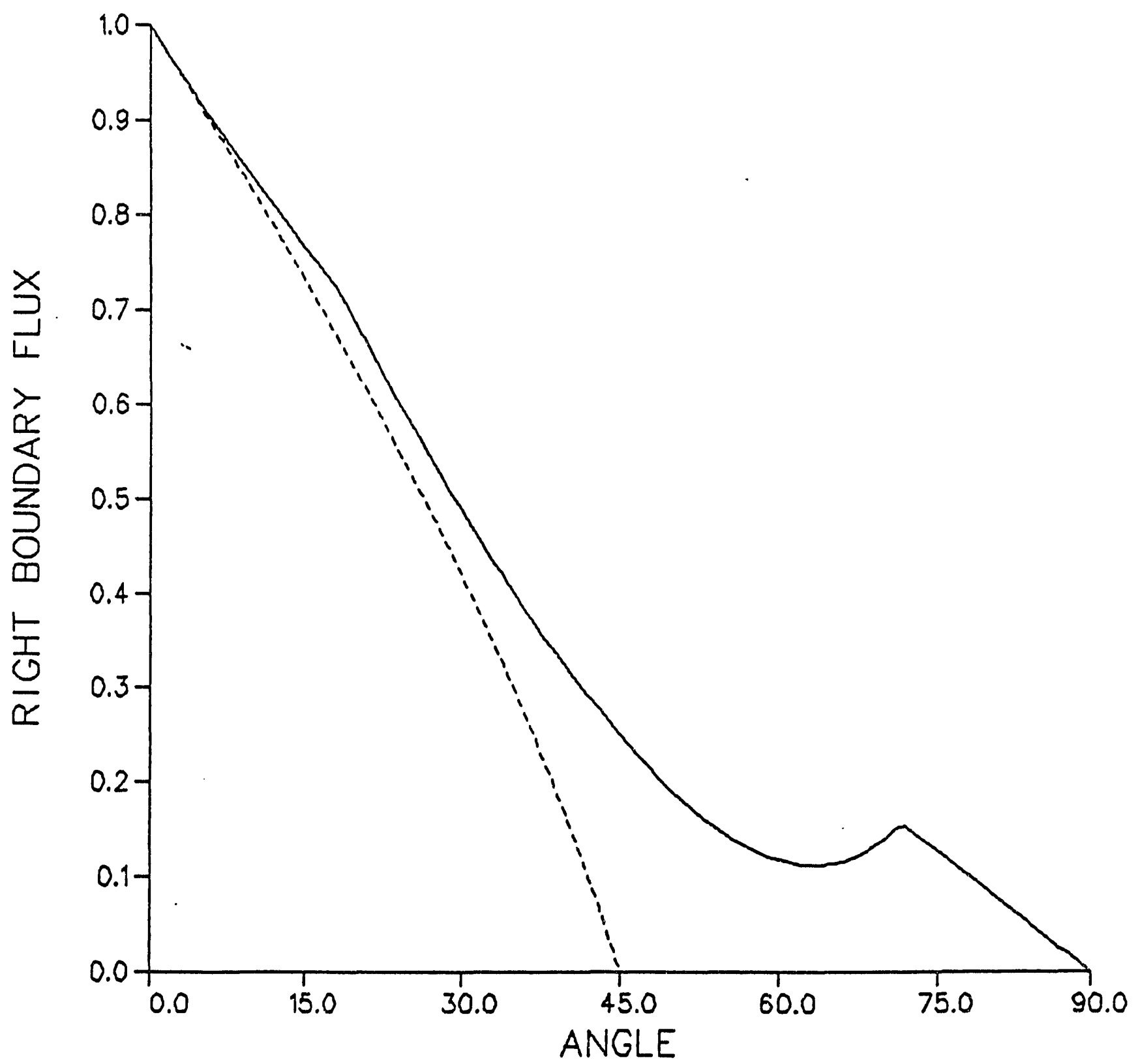

Figure 4.6 The Linear Nodal Solution With the Step Limit. 


\section{NEGATIVE FLUX PREVENTION}

In order to find conditions sufficient to guarantee that the linear nodal method will not yield negative fluxes given positive sources, substitute the expression for $\psi_{a v}$ (Equation (3.9)) into the expressions for $\psi_{R}, \psi_{\text {out }}$, and $\psi_{T}$ (Equations (3.7)) to obtain

$$
\begin{aligned}
& \psi_{R}=\frac{\gamma_{x}}{D}\left(S_{a v}+T_{x} \psi_{L}+\beta_{y} \psi_{\text {in }}+\beta_{z} \psi_{B}+g_{x} Q^{x}-\alpha_{y} Q^{y}-\alpha_{z} Q^{z}\right) \\
& \psi_{\text {out }}=\frac{\gamma_{y}}{D}\left(S_{a v}+\beta_{x} \psi_{L}+T_{y} \psi_{\text {in }}+\beta_{z} \psi_{B}-\alpha_{x} Q^{x}+g_{y} Q^{y}-\alpha_{z} Q^{z}\right),
\end{aligned}
$$

and

$$
\psi_{T}=\frac{\gamma_{z}}{D}\left(S_{a v}+\beta_{x} \psi_{L}+\beta_{y} \psi_{\mathrm{in}}+T_{z} \psi_{B}-\alpha_{x} Q^{x}-\alpha_{y} Q^{y}+g_{z} Q^{z}\right)
$$

$$
\text { where } \begin{aligned}
T_{x} & =\beta_{x}-D\left(1-\frac{1}{\gamma_{x}}\right) \\
T_{y} & =\beta_{y}-D\left(1-\frac{1}{\gamma_{y}}\right) \\
T_{z} & =\beta_{z}-D\left(1-\frac{1}{\gamma_{z}}\right) \\
g_{x} & =\frac{\alpha_{x}}{\beta_{x}}\left(D-\beta_{x}\right)=\frac{\alpha_{x}}{\beta_{x}}\left(\sigma+\beta_{y}+\beta_{z}\right), \\
g_{y} & =\frac{\alpha_{y}}{\beta_{y}}\left(D-\beta_{y}\right)=\frac{\alpha_{y}}{\beta_{y}}\left(\sigma+\beta_{x}+\beta_{z}\right), \\
\text { and } \quad g_{z} & =\frac{\alpha_{z}}{\beta_{z}}\left(D-\beta_{z}\right)=\frac{\alpha_{z}}{\beta_{z}}\left(\sigma+\beta_{x}+\beta_{y}\right) .
\end{aligned}
$$

All of the coefficients in Equations (5.1) are positive quantities. This can be seen immediately from the definition for all of the coefficients except $T_{x}, T_{y}$, and $T_{z}$, and numerical tests indicate that $T_{x}, T_{y}$, and $T_{z}$ are always positive also. For the one-dimensional case, the expression for $T_{x}$ simplifies after considerable algebra to 


$$
T_{x}=\frac{D}{\gamma_{x}} e^{-\epsilon_{x}}
$$

This illustrates that $T_{x}$ is indeed always positive in this case.

Substituting the expressions for $Q^{x}, Q^{y}$, and $Q^{z}$ (Equations (3.3) and (3.8)) into the equations for $\psi_{a v}, \psi_{R}, \psi_{\text {out }}$, and $\psi_{T}$ (Equations (3.9) and (5.1)) and grouping terms yields

$$
\begin{aligned}
\psi_{a v}=\frac{1}{D}( & S_{a v}-\alpha_{x} S^{x}-\alpha_{y} S^{y}-\alpha_{z} S^{z} \\
& +\beta_{x} \psi_{L}-\rho_{x} \alpha_{y} \psi_{L}^{y}-\rho_{x} \alpha_{z} \psi_{L}^{z} \\
& +\beta_{y} \psi_{\mathrm{in}}-\rho_{y} \alpha_{x} \psi_{\mathrm{in}}^{x}-\rho_{y} \alpha_{z} \psi_{\mathrm{in}}^{z} \\
& \left.+\beta_{z} \psi_{B}-\rho_{z} \alpha_{x} \psi_{B}^{x}-\rho_{z} \alpha_{y} \psi_{B}^{y}\right) \\
\psi_{R}=\frac{\gamma_{x}}{D}( & S_{\mathrm{a} v}+g_{x} S^{x}-\alpha_{y} S^{y}-\alpha_{z} S_{z} \\
& +T_{x} \psi_{L}-\rho_{x} \alpha_{y} \psi_{L}^{y}-\rho_{x} \alpha_{z} \psi_{L}^{z} \\
& +\beta_{y} \psi_{\mathrm{in}}+\rho_{y} g_{x} \psi_{\mathrm{in}}^{x}-\rho_{y} \alpha_{z} \psi_{\mathrm{in}}^{z} \\
& \left.+\beta_{z} \psi_{B}+\rho_{z} g_{x} \psi_{B}^{x}-\rho_{z} \alpha_{y} \psi_{B}^{y}\right) \\
\psi_{\mathrm{out}}=\frac{\gamma_{y}}{D}( & S_{a v}-\alpha_{x} S^{x}+g_{y} S^{y}-\alpha_{z} S^{z} \\
& +\beta_{x} \psi_{L}+\rho_{x} g_{y} \psi_{L}^{y}-\rho_{x} \alpha_{z} \psi_{L}^{z} \\
& +T_{y} \psi_{\mathrm{in}}-\rho_{y} \alpha_{x} \psi_{\mathrm{in}}^{x}-\rho_{y} \alpha_{z} \psi_{\mathrm{in}}^{z} \\
& \left.+\beta_{z} \psi_{B}-\rho_{z} \alpha_{x} \psi_{B}^{x}+\rho_{z} g_{y} \psi_{B}^{y}\right)
\end{aligned}
$$

and

$$
\begin{aligned}
\psi_{T}=\frac{\gamma_{z}}{D}( & S_{a v}-\alpha_{x} S^{x}-\alpha_{y} S^{y}+g_{z} S^{z} \\
& +\beta_{x} \psi_{L}-\rho_{x} \alpha_{y} \psi_{L}^{y}+\rho_{x} g_{z} \psi_{L}^{z} \\
& +\beta_{y} \psi_{\mathrm{in}}-\rho_{y} \alpha_{x} \psi_{\mathrm{in}}^{x}+\rho_{y} g_{z} \psi_{\mathrm{in}}^{z} \\
& \left.+T_{z} \psi_{B}-\rho_{z} \alpha_{x} \psi_{B}^{x}-\rho_{z} \alpha_{y} \psi_{B}^{y}\right)
\end{aligned}
$$

The terms in Equations (5.2) which contain $S, S^{x}, S^{y}$, and $S^{z}$ represent the contribution of $S(x, y, z)$ to the four average values $\psi_{a v}, \psi_{R}, \psi_{\text {in }}$, and $\psi_{T}$. The 
following four conditions are required to insure that the contributions of $S(x, y, z)$ in Equations (5.2) are non-negative:

$$
\begin{aligned}
& S_{a v}-\alpha_{x} S^{x}-\alpha_{y} S^{y}-\alpha_{z} S^{z} \geq 0 \\
& S_{a v}+g_{x} S^{x}-\alpha_{y} S^{y}-\alpha_{z} S^{z} \geq 0 \\
& S_{a v}-\alpha_{x} S^{x}+g_{y} S^{y}-\alpha_{z} S^{z} \geq 0 \\
& S_{a v}-\alpha_{x} S^{x}-\alpha_{y} S^{y}+g_{z} S^{z} \geq 0
\end{aligned}
$$

Sufficient adjusted values of $S^{x}, S^{y}$, and $S^{z}$ to satisfy Equation (5.3a) are

$$
\begin{aligned}
& S_{1}^{x}=\min \left(S^{x}, \frac{S_{a v}}{3 \alpha_{x}}\right) \\
& S_{1}^{y}=\min \left(S^{y}, \frac{S_{a v}}{3 \alpha_{y}}\right)
\end{aligned}
$$

and

$$
S_{1}^{z}=\min \left(S^{z}, \frac{S_{a v}}{3 \alpha_{z}}\right)
$$

where the 1 subscript indicates the adjusted value. For example, $S_{1}^{x}$ will be no larger than $\frac{S_{a v}}{3 \alpha_{x}}$ no matter how large $S^{x}$ is. From Equation (3.6), it is easily shown that

$$
\alpha_{x} \leq \frac{1}{3}
$$

It follows that

$$
\frac{S_{a v}}{3 \alpha_{x}} \geq S_{a v}
$$


Waiters suggests an upper bound on $S^{x}$ of $S_{a v}$, so the upper bound of $\frac{S_{a v}}{3 \alpha_{x}}$ is less restrictive than Walters' bound.

From Equation (5.3b), it can be seen that a lower bound on $S^{x}$ is

$$
S^{x} \geq-\frac{1}{g_{x}}\left(S_{a v}-\alpha_{y} S^{y}-\alpha_{z} S^{z}\right)
$$

Numerial testing shows that

$$
\frac{1}{g_{x}} \geq 1
$$

Walters lower limit for $S^{x}$ is $-S_{a v}$, which is similar to Equation (5.5) except that positive values for $S^{y}$ and/or $S^{z}$ could result in a negative value for $\psi_{R}$ even when Walters' criterion is met. Expressions for $S^{x}, S^{y}$, and $S^{z}$ which satisfy all of the criteria in Equations (5.3) and are also easily calculated without complicated logic are

$$
\begin{aligned}
& S_{2}^{x}=\max \left(S_{1}^{x},-\frac{S_{E}}{g_{x}}\right) \\
& S_{2}^{y}=\max \left(S_{1}^{y},-\frac{S_{E}}{g_{y}}\right) \\
& S_{2}^{z}=\max \left(S_{1}^{z},-\frac{S_{E}}{g_{z}}\right)
\end{aligned}
$$

where

$$
S_{E}=S_{a v}-\alpha_{x} \min \left(S_{1}^{x}, 0\right)-\alpha_{y} \min \left(S_{1}^{y}, 0\right)-\alpha_{z} \min \left(S_{1}^{z}, 0\right)
$$

Note that the worst case in terms of a negative value for $\psi_{R}$ is $S^{x}<0, S^{y}>0$, and $S^{z}>0$. For this case

$$
S_{E}=S_{a v}-\alpha_{y} S_{1}^{y}-\alpha_{z} S_{1}^{z}=S_{a v}-\alpha_{y} S_{2}^{y}-\alpha_{z} S_{2}^{z}
$$


and the inequality in Equation (5.5) is satisfied by $S_{2}^{x}, S_{2}^{y}$, and $S_{2}^{z}$.

The terms in Equations (5.2) which contain $\psi_{L}, \psi_{L}^{y}$, and $\psi_{L}^{z}$ represent the contribution of $\psi_{L}(y, z)$ to the four average values $\psi_{a v}, \psi_{R}, \psi_{\text {out }}$, and $\psi_{T}$. The following four conditions are required to insure that the contribution of $\psi_{L}(y, z)$ in Equations (5.2) is non-negative:

$$
\begin{aligned}
& \beta_{x} \psi_{L}-\rho_{x} \alpha_{y} \psi_{L}^{y}-\rho_{x} \alpha_{z} \psi_{L}^{z} \geq 0 \\
& T_{x} \psi_{L}-\rho_{x} \alpha_{y} \psi_{L}^{y}-\rho_{x} \alpha_{z} \psi_{L}^{z} \geq 0 \\
& \beta_{x} \psi_{L}+\rho_{x} g_{y} \psi_{L}^{y}-\rho_{x} \alpha_{z} \psi_{L}^{z} \geq 0
\end{aligned}
$$

and

$$
\beta_{x} \psi_{L}-\rho_{x} \alpha_{y} \psi_{L}^{y}+\rho_{x} g_{z} \psi_{L}^{z} \geq 0
$$

'The definition of $T_{x}$ is

$$
T_{x}=\beta_{x}-D\left(1-\frac{1}{\gamma_{x}}\right)
$$

and the step limit corrrection insures that

$$
\gamma_{x} \geq 1
$$

Thus, it is always the case that

$$
T_{x} \leq \beta_{x}
$$

and that Equation (5.7a) will always be satisfied if Equation (5.7b) is satisfied. The following conditions are sufficient to satisfy Equations (5.7): 


$$
\begin{aligned}
\frac{T_{x}}{2} \psi_{L}-\rho_{x} \alpha_{y} \psi_{L}^{y} & \geq 0 \\
\frac{T_{x}}{2} \psi_{L}-\rho_{x} \alpha_{z} \psi_{L}^{z} & \geq 0 \\
\frac{\beta_{x}}{2} \psi_{L}+\rho_{x} g_{y} \psi_{L}^{y} & \geq 0 \\
\text { and } \frac{\beta_{x}}{2} \psi_{L}+\rho_{x} g_{z} \psi_{L}^{z} & \geq 0
\end{aligned}
$$

Thus, sufficient upper and lower bounds for $\psi_{L}^{y}$ are

$$
-\frac{1}{2} \frac{\beta_{x}}{\rho_{x} g_{y}} \psi_{L} \leq \psi_{L}^{y} \leq \frac{1}{2} \frac{T_{x}}{\rho_{x} \alpha_{y}} \psi_{L}
$$

For $\psi_{L}^{z}$ the bounds are

$$
-\frac{1}{2} \frac{\beta_{x}}{\rho_{x} g_{z}} \psi_{L} \leq \psi_{L}^{z} \leq \frac{1}{2} \frac{T_{x}}{\rho_{x} \alpha_{z}} \psi_{L}
$$

Numerial testing has indicated that the use of the limits is equations (5.8) and their analogs for $\psi_{\mathrm{in}}^{x}, \psi_{\mathrm{in}}^{z}, \psi_{B}^{x}$, and $\psi_{B}^{y}$ reduce the accuracy of the results. It has also been found that relaxing the bounds by removing the " $\frac{1}{2}$ " such that

$$
-\frac{\beta_{x}}{\rho_{x} g_{y}} \psi_{L} \leq \psi_{L}^{y} \leq \frac{T_{x}}{\rho_{x} \alpha_{y}} \psi_{L}
$$

improves the accuracy with no apparent difficulty for some problems. For other problems, negative fluxes are observed when the relaxed bounds are used.

One difficulty with this correction involves the terms $T_{x}, T_{y}$, and $T_{z}$. For a single mesh cell and angle with a flat boundary flux on the left boundary, the result for $\psi_{R}$ is

$$
\psi_{R}=\frac{\gamma_{x}}{D} T_{x} \psi_{L}
$$

As was seen in section 4 , the correct value for $\psi_{R}$ in this case is often zero. However, the linear nodal equations always yield a positive value for $\psi_{R}$ which is not correct physically. In these cases, limiting the values of $\psi_{L}^{y}$ and $\psi_{L}^{z}$ to prevent $\psi_{R}$ from 
becoming negative, when in fact $\psi_{R}$ should be zero no matter what $\psi_{L}^{y}$ and $\psi_{L}^{z}$ are is also not correct physically and could be responsible for the reduced accuracy.

Other lower limits for $\psi_{L}^{y}$ and $\psi_{L}^{z}$ can be obtained from Equations(5.7c) and (5.7d). These are

$$
\begin{gathered}
\psi_{L}^{y} \geq-\left(\frac{\beta_{x}}{\rho_{x} g_{y}} \psi_{L}-\frac{\alpha_{z}}{g_{y}} \psi_{L}^{z}\right) \\
\text { and } \psi_{L}^{z} \geq-\left(\frac{\beta_{x}}{\rho_{x} g_{z}} \psi_{L}-\frac{\alpha_{y}}{g_{z}} \psi_{L}^{y}\right) .
\end{gathered}
$$

a simple set of bounds which satisfy Equations (5.10) are

$$
\begin{gathered}
\psi_{L}^{y} \geq-\left[\frac{\beta_{x}}{\rho_{x} g_{y}} \psi_{L}-\frac{\alpha_{z}}{g_{y}} \min \left(\psi_{L}^{z}, 0\right)\right] \\
\text { and } \psi_{L}^{z} \geq-\left[\frac{\beta_{x}}{\rho_{x} g_{z}} \psi_{L}-\frac{\alpha_{y}}{g_{z}} \min \left(\psi_{L}^{y}, 0\right)\right]
\end{gathered}
$$

These alternative lower bounds may improve accuracy while maintaining positive results, but they have not been tested extensively.

Numerical studies have been performed to obtain limits on the coefficients in Equation (5.9).

These are

$$
\begin{aligned}
\frac{T_{x}}{\rho_{x} \alpha_{y}} & \geq \frac{2}{3} \\
\text { and } \frac{\beta_{x}}{\rho_{x} g_{y}} & \geq \frac{1}{2} .
\end{aligned}
$$

Both of these limits are non-physical $m$ that values less than one indicate that an all positive incoming boundary flux distribution can cause negative fluxes. 


\section{IMPLEMENTATION IN TORT}

In order to perform linear nodal calculations using the equations developed in previous sections, the functions $F(x)$ and $G(x)$ introduced in sections 2 and 3 must be evaluated. These functions are defined in terms of exponentials and can be evaluated directly from their definitions when $x$ is not near zero. Walters suggests using the approximation

$$
e^{-x}=\frac{60-24 x+3 x^{2}}{60+36 x+9 x^{2}+x^{3}}
$$

When this approximation is used

$$
F(x)=\frac{60+6 x+x^{2}}{30+8 x+x^{2}}
$$

and

$$
G(x)=\frac{5+x}{10+x}
$$

The maximum error in the approximation for $F(x)$ is about 6.7 percent. A result similar to Equation (6.2) with a maximum error of about 0.5 percent is

$$
F(x)=\frac{17.6767+4.27049 x+x^{2}}{8.83835+3.52633 x+x^{2}}
$$

Equation (6.4) is used in TORT; it preserves the two limits $F(0)=2$ and $F(\infty)=1$ exactly and is quite accurate for all other values.

Equation (6.3) has a maximum error of about 10 percent. A much more accurate expression for $G(x)$ is

$$
G(x)=\frac{15.1+4.53 x+x^{2}}{30.2+6.53 x+x^{2}}
$$

This result is based of the following always positive approximation: 


$$
e^{-x}=\frac{181.2-51.42 x+4.04 x^{2}}{181.2+129.78 x+43.22 x^{2}+8.53 x^{3}+x^{4}}
$$

Equation (6.6) is more accurate than Equation (6.1). It is important that the approximation for $G(x)$ is based on an approximation for $e^{-x}$ that is positive for all values of $x$. An approximation for $G(x)$ used earlier in TORT was based on a direct fit to $G(x)$ which had a maximum error of 0.3 percent and resulted in negative results in rare cases.

Another extension of the linear nodal method is required to treat anisotropic scattering. An easy way to correct for this is to assume the spatial distribution for $S(x, y, z)$ is separable from the angular distribution due to the $P_{L}$ expansion of the scattering cross section. This is implemented by simply multiplying $S_{a v}, S^{x}, S^{y}$, and $S^{z}$ by the ratio $\left(S_{\text {anisotropic }} / S_{\text {isotropic }}\right)$ where the ratio can be viewed as a correction factor relating the value of the anistropic source to the source obtained when only the scalar flux is considered. This approximation may not always be desirable, however. For example, K-effective calculations can be affected adversely.

The equations derived earlier are based on a coordinate system in which the direction cosines of the discrete direction are all positive. Since some direction cosines are negative, $a$ simple coordinate system transformation is required. For example, if $\mu<0$, the sign of $S^{x}$ must be changed. The sign of $\psi^{x}$ must also be changed in this case. Similar changes are made when $\xi$ or $\eta$ are negative.

The calculation of the scalar scattering and fission sources for the linear nodal method include $x, y$, and $z$ flux moments as well as the scalar flux moment. For this reason, $a P_{3}$ calculation with weighted difference has 16 flux moments, while a $P_{3}$ linear nodal calculation has 19 . The three additional moments are treated in exactly the same manner as the scalar flux to obtain the source. For example, if

$$
\begin{aligned}
S_{a v}(g) & =\sum_{g^{\prime}} \sigma\left(g^{\prime} \rightarrow g\right) \psi_{a v}\left(g^{\prime}\right) \text { then } \\
S^{x}(g) & =\sum_{g^{\prime}} \sigma\left(g^{\prime} \rightarrow g\right) \psi^{x}\left(g^{\prime}\right) .
\end{aligned}
$$




\section{THE LINEAR CHARACTERISTIC METHOD}

In the following development it is convenient to introduce a local coordinate system for the space cell in which

$$
\begin{aligned}
& 0 \leq x \leq \Delta x, \\
& 0 \leq y \leq \Delta y, \\
\text { and } \quad & 0 \leq z \leq \Delta z .
\end{aligned}
$$

Otherwise, the notation is the same as that used previously. A useful quantity to introduce is

$$
A_{u} \equiv \max \left(A_{x}, A_{y}, A_{z}\right)
$$

The physical significance of $A_{u}$ is found by considering

$$
d_{\max } \equiv \frac{1}{A_{u}}=\min \left(\frac{1}{A_{x}}, \frac{1}{A_{y}}, \frac{1}{A_{z}}\right)=\min \left(\frac{\Delta x}{\mu}, \frac{\Delta y}{\xi}, \frac{\Delta z}{\eta}\right) .
$$

Thus, $d_{\max }$ is the distance along the discrete direction from position $(0,0,0)$ to the boundary of the cell. Also, $d_{\max }$ is the distance backwards along the discrete direction from position $(\Delta x, \Delta y, \Delta z)$ to the cell boundary. $d_{\max }$ is the maximum distance a particle can travel in the cell without a collision along the discrete direction.

Next consider the parametric equations for the line starting at position $(0,0,0)$ along the discrete direction

$$
\begin{aligned}
x & =\mu d, \\
y & =\xi d, \\
\text { and } z & =\eta d,
\end{aligned}
$$

where $d$ is the distance from point $(0,0,0)$ to any point $(x, y, z)$ on the line. An alternative form of these equations is 


$$
\begin{aligned}
\frac{x}{\Delta x} & =A_{x} d, \\
\frac{y}{\Delta y} & =A_{y} d, \\
\text { and } \frac{z}{\Delta z} & =A_{z} d .
\end{aligned}
$$

Replacing the distance $d$ with a fractional distance $u$ defined by

$$
u \equiv \frac{d}{d_{\max }}
$$

results in

$$
\begin{aligned}
\frac{x}{\Delta x} & =w_{x} u \\
\frac{y}{\Delta y} & =w_{y} u \\
\text { and } \frac{z}{\Delta z} & =w_{z} u \\
\text { where } \quad w_{x} & =\frac{A_{x}}{A_{u}} \\
w_{y} & =\frac{A_{y}}{A_{u}}, \\
\text { and } \quad w_{x} & =\frac{A_{z}}{A_{u}} .
\end{aligned}
$$

Notice that $w_{x}, w_{y}$, and $w_{z}$ are simply $A_{x}, A_{y}$, and $A_{z}$ normalized such that the largest one is unity. When $u=1$, the boundary crossing point is $\left(w_{x} \Delta x, w_{y} \Delta y, w_{z} \Delta z\right)$. This information can be used to determine which portion of incoming boundary fluxes contribute to the average angular flux on a given outgoing face. Figure 7.1 illustrate this point for two-dimensional $x-z$ geometry. In each case, line segment $A B$ is along the discrete direction. Any particle entering the cell on the left or bottom boundary and traveling along the discrete direction will follow a straight line parallel to $A B$ until it has a collision or reaches the cell boundary. Any particle that leaks without collision will leak out the top boundary if its path is above $A B$. Similarly, any paths below $A B$ will leak out the right boundary. The special case of particles leaking out the corner rather than either face is not important unless a delta function is allowed in the incoming boundary 
Case I

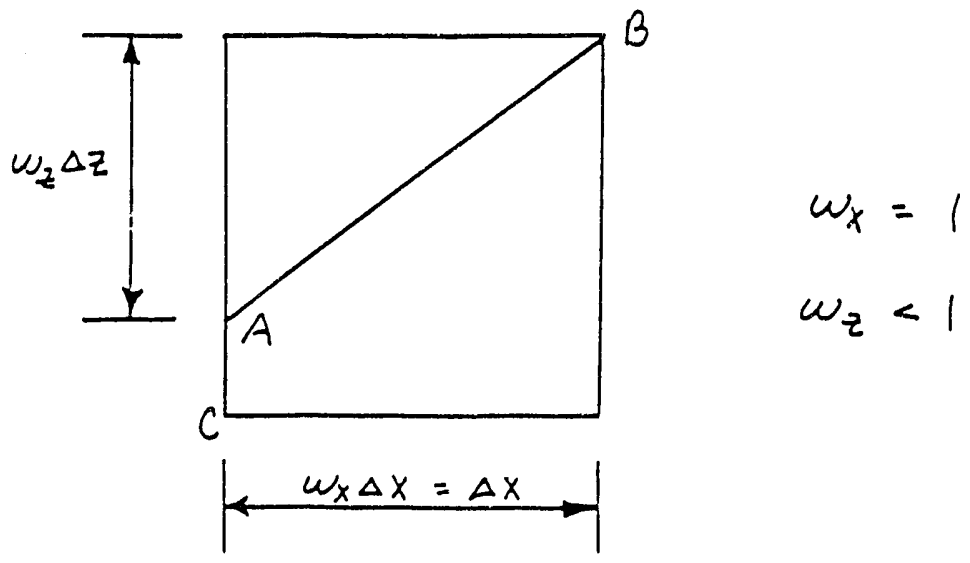

case II

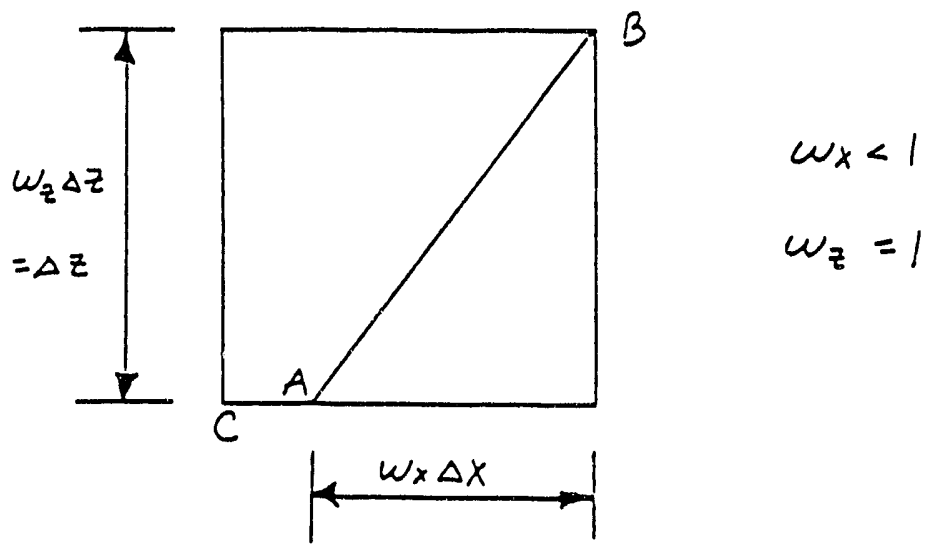

Case III

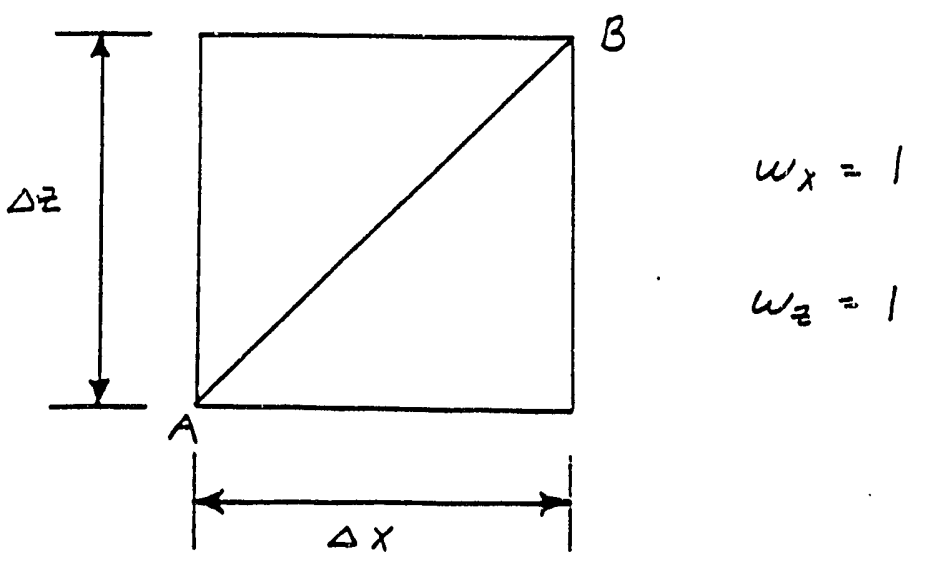

Figure 7.1 Two Dimensional Cases For The Characteristic Method. 
flux. Notice that the particle's path-length when it enters at $A$ and exits at $B$ is $d_{\max }$. Also notice that any particle that enters along line segement $A C$ also travels distance $d_{\max }$ before exiting without collision.

Next consider the more complicated three-dimensional case in which the incoming faces are two dimensional. Figure 7.2 shows different cases for particles entering the $i n$ face at $y=0$ as a function of $x$ and $z$. These cases illustrate where a particle entering the boundary will leak if it travels along the discrete direction without collision. One iusportant point is that although four cases are shown, cases II-IV are just special cases of case $I$ in which $w_{x}, w_{z}$, or both equal unity. Also, the distance along the discrete direction from $A$ to the cell boundary is $d_{\max }$ in all four cases.

The next step in the derivation is to obtain exact expressions for $\psi_{\vec{\zeta}}, \psi_{\Gamma}$, and $\psi_{\text {out }}$ that result from the uncollided transport of $\psi_{\text {in }}(x, z)$ through the cell. These are obtained by integrating over the appropriate : ea in Figure 7.2. For $1 / \mathrm{s}_{\mathrm{u}}$, the expression is

$$
\begin{gathered}
\psi_{\text {'out }} \Delta x \Delta z=\int_{0}^{\Delta z\left(1-w_{z}\right)} \int_{0}^{\Delta x\left(1-w_{r}\right)} e^{-\epsilon} \psi_{\text {in }}(x, z) d x d z \\
\text { where } \epsilon=\sigma d_{\max }=\frac{\sigma}{A_{u}}=\min \left(\dot{z}_{x}, \epsilon_{y}, \epsilon_{z}\right) .
\end{gathered}
$$

In order to integrate over the area indicated in Figure 7.2 for $\psi_{T}$, parametric equations for line segment $B A$ is useful. These are

$$
x=\Delta x\left(1-w_{x} u\right)
$$

$$
\text { and } z=\Delta z\left(1-w_{z} u\right)
$$

Notice that when $u=0$, the location is point $B,(\Delta x, \Delta z)$, while $u=1$ corresponds to point $A,\left(\Delta x-w_{x} \Delta x, \Delta z-w_{z} \Delta z\right)$.

The equation for $\psi_{T}$ is

$$
\eta \psi_{T} \Delta x \Delta y=\int_{\Delta z\left(1-w_{z}\right)}^{\Delta z} e^{-\epsilon u} \int_{0}^{\Delta x\left(1-w_{x} u\right)} \xi \psi_{\mathrm{in}}(x, z) d x d z
$$


Case I
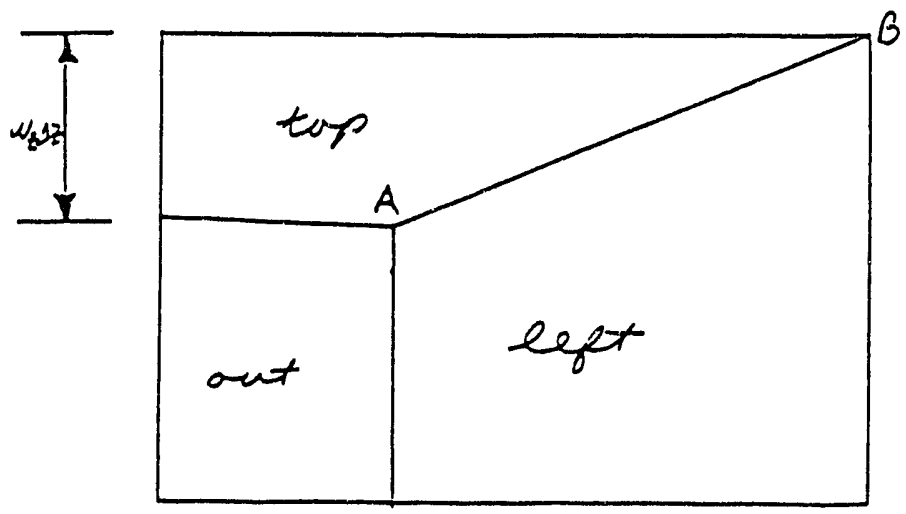

$$
\begin{aligned}
& w_{x}<1 \\
& w_{y}=1 \\
& w_{z}<1
\end{aligned}
$$

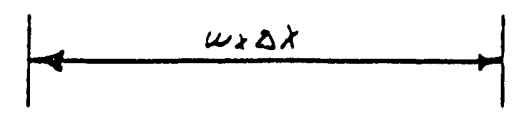

Case I
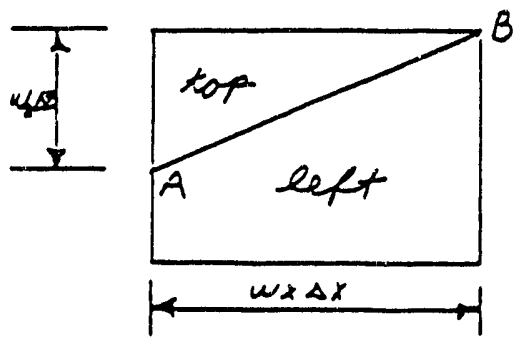

$$
\begin{aligned}
& w_{x}=1 \\
& w_{z}<1
\end{aligned}
$$

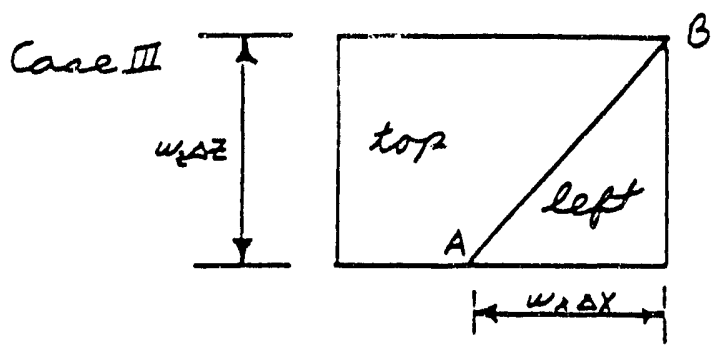

$$
\begin{aligned}
& w_{x}<1 \\
& w_{z}=1
\end{aligned}
$$
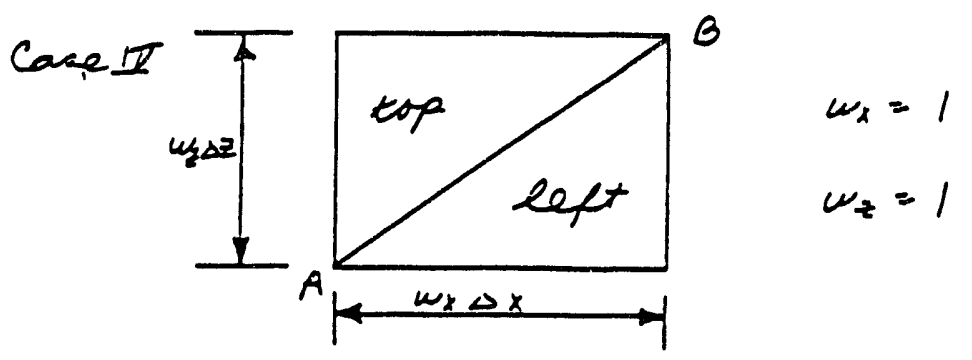

Figure 7.2 Leakage Surface by Location on the $y=0$ Cell Face.

36 
This equation makes use of the fact that the distance traveled from points on $A B$ along the discrete direction to the cell boundary is proportional to $u$, and this distance is the same for all points at that $z . u$ can be considered $u(z)$ and obtained from Equation (7.5b). The equation for $\psi_{R}$ is entirely analogous to Equation (7.6).

In order to evaluate Equations (7.4) and (7.6) for $\psi_{\text {out }}$ and $\psi_{T}$ the following expression for $\psi_{\text {in }}(x, z)$ is introduced:

$$
\begin{gathered}
\psi_{\mathrm{in}}(x, z)=\left[\left(1-\tilde{\psi}_{\mathrm{in}}^{x}\right)+\tilde{\psi}_{\mathrm{in}}^{x} \frac{2 x}{\Delta x}\right]\left[\left(1-\tilde{\psi}_{\mathrm{in}}^{z}\right)+\tilde{\psi}_{\mathrm{in}}^{z} \frac{2 z}{\Delta z}\right] \psi_{\mathrm{in}}, \\
\text { where } \quad \tilde{\psi}_{\mathrm{in}}^{x}=\frac{\psi_{\mathrm{in}}^{x}}{\psi_{\mathrm{in}}} \\
\text { and } \quad \tilde{\psi}_{\mathrm{in}}^{z}=\frac{\psi_{\mathrm{in}}^{z}}{\psi_{\mathrm{in}}}
\end{gathered}
$$

This expression represents $\psi(x, z)$ as the separable product of a linear expansion in $x$ and a linear expansion in $z$. The modivation for choosing this form is that it is very easy to insure that

$$
\psi_{\text {in }}(x, z) \geq 0
$$

for all points on the cell face.

These conditions are

$$
\begin{aligned}
& \left|\tilde{\psi}_{\text {in }}^{x}\right| \leq 1 \quad \text { and } \\
& \left|\tilde{\psi}_{\text {in }}^{z}\right| \leq 1 .
\end{aligned}
$$

From this point forward in the derivation the " $"$ will be dropped to simplify notation.

A useful expression for evaluating integrals of linear expansions is

$$
\frac{1}{\Delta x} \int_{0}^{\Delta x\left(1-u w_{x}\right)}\left[\left(1-\psi_{\mathrm{in}}^{x}\right)+\psi_{\mathrm{in}}^{x} \frac{2 x}{\Delta x}\right] d x=\left(1-u w_{x}\right)\left(1-u w_{z} \psi_{\mathrm{in}}^{x}\right)
$$

Substituting Equation (7.7) into Equation (7.4) and evaluating the two integrals yields 


$$
\psi_{\mathrm{out}}=\psi_{\mathrm{in}} e^{-\epsilon}\left(1-w_{x}\right)\left(1-w_{z}\right)\left(1-w_{x} \psi_{\mathrm{in}}^{x}\right)\left(1-w_{z} \psi_{\mathrm{in}}^{z}\right)
$$

Similarly substituting Equation (7.7) into Equation (7.6) and evaluating the integral over $x$ yields

$$
\psi_{T}=A_{y} \psi_{\mathrm{in}} \frac{1}{\eta} \int_{\Delta z\left(1-w_{x}\right)}^{\Delta z} e^{-\epsilon u}\left[\left(1-\psi_{\mathrm{in}}^{z}\right)+\psi_{\mathrm{in}}^{z} \frac{2 z}{\Delta z}\right]\left(1-u w_{x}\right)\left(1-u w_{x} \psi_{\mathrm{in}}^{x}\right) d z
$$

A much better form of Equation (7.10) is obtained by a change of variable from $z$ to $u$. From Equation ( $7.5 \mathrm{~b})$, it is noted that

$$
d z=-w_{z} \Delta z d u
$$

After the change of variable, Equation (7.10) becomes

$$
\psi_{T}=w_{y} \psi_{\mathrm{in}} \int_{0}^{1} e^{-\epsilon u}\left[\left(1+\psi_{\mathrm{in}}^{z}\right)-2 w_{z} \psi_{\mathrm{in}}^{z} u\right]\left(1-w_{x} u\right)\left(1-w_{x} \psi_{\mathrm{in}}^{x} u\right) d u
$$

The solution to this equation can be written in terms of

$$
F_{n}(\epsilon) \equiv \int_{0}^{1} u^{n-1} e^{-\epsilon u} d u, n>0
$$

Since this derviation is for a single mesh cell, the dependence on $\varepsilon$ can be dropped in the notation without ambiguity. While the solution can be written in terms of $F_{n}$, it is convenient to introduce an additional quantity

$$
G_{n}^{x} \equiv \int_{0}^{1} u^{n-2} e^{-\epsilon u}\left(1-w_{x} u\right) d u=F_{n-1}-w_{x} F_{n}, n>1
$$

The solution to Equation (7.11) is

$$
\begin{array}{r}
\psi_{T}=w_{y} \psi_{\mathrm{in}}\left[\left(1+\psi_{\mathrm{in}}^{z}\right)\left(G_{2}^{x}-G_{3}^{x} w_{x} \psi_{\mathrm{in}}^{x}\right)\right. \\
\left.-2 w_{z} \psi_{\mathrm{in}}^{z}\left(G_{3}^{x}-G_{4}^{x} w_{x} \psi_{\mathrm{in}}^{x}\right)\right] .
\end{array}
$$


The analogous equation for $\psi_{R}$ is

$$
\begin{aligned}
\psi_{R}=w_{y} \psi_{\mathrm{in}} & \left(1+\psi_{\mathrm{in}}^{x}\right)\left(G_{2}^{z}-G_{3}^{z} w_{z} \psi_{\mathrm{in}}^{z}\right. \\
& \left.-2 w_{x} \psi_{\mathrm{in}}^{x}\left(G_{3}^{z}-G_{4}^{z} w_{z} \psi_{\mathrm{in}}^{z}\right)\right]
\end{aligned}
$$

In addition to $\psi_{R}, \psi_{\text {out }}$, and $\psi_{T}$, an expression for $\psi_{a v}$ is needed. An integral equation for $\psi_{a v}$ can be formulated, but the approach used here will be to use the particle balance equation. Dividing Equation (2.2) by $A_{u}$ yields a convenient form of the balance equation:

$$
\epsilon \psi_{a v}=\frac{S_{a v}}{A_{u}}+w_{x}\left(\psi_{L}-\psi_{R}\right)+w_{y}\left(\psi_{\text {in }}-\psi_{\text {out }}\right)+w_{z}\left(\psi_{B}-\psi_{T}\right)
$$

For the special case of a boundary flux on the incoming face only, Equation (7.16) becomes

$$
\epsilon \psi_{a v}=w_{y} \psi_{\text {in }}-\left(w_{x} \psi_{R}+w_{y} \psi_{\text {out }}+w_{z} \psi_{T}\right)
$$

Equation (7.16) can be used to obtain $\psi_{a v}$ when $\epsilon$ is not small or zero. However, another expression is needed for small $\epsilon$. This is obtained by substituting Equations (7.9), (7.14), and (7.15) into Equation (7.17). The remaining problem is how to handle the required division by $\epsilon$. The function $F_{1}$ is given by

$$
F_{1} \equiv \int_{0}^{1} e^{-\epsilon u} d u=\frac{1-e^{-\epsilon}}{\epsilon}
$$

This expression allows $\frac{1-e^{-\epsilon}}{\epsilon}$ to be replaced by $F_{1}$ in the derivation. $F_{1}$ approaches 1 as $\epsilon$ approaches 0 . Another useful expression is obtained by integrating Equation (7.12) by parts. This is

$$
F_{n+1}=\frac{n F_{n}-e^{-\epsilon}}{\epsilon}, n>0
$$

using Equation (7.18) to eliminate reference to $e^{-\epsilon}$ in Equation (7.19) yields 


$$
\frac{1-n F_{n}}{\epsilon}=F_{1}-F_{n+1} \text {. }
$$

Using Equations (7.18) and (7.20) and lengthy but straighforward algebra, the following expression for $\psi_{a v}$ is obtained:

$$
\begin{gathered}
\qquad \begin{array}{c}
\psi_{a v}=w_{y} \psi_{\mathrm{in}}\left[\left(H_{3}^{y}-w_{x} \psi_{\mathrm{in}}^{x} H_{4}^{y}\right)\right. \\
\left.-w_{z} \psi_{\mathrm{in}}^{z}\left(H_{4}^{y}-w_{x} \psi_{\mathrm{in}}^{x} H_{5}^{y}\right)\right], \\
\text { where } H_{n}^{y} \equiv \int_{0}^{1} u^{n-3} e^{-\epsilon u}\left(1-w_{x} u\right)\left(1-w_{z} u\right) d u \\
=F_{n-2}-F_{n-1}\left(w_{x}+w_{z}\right)+F_{n} w_{x} w_{z}, n>2 ;
\end{array}
\end{gathered}
$$

and this is valid for all $\epsilon$, zero or not.

In addition to the equations for $\psi_{R}, \psi_{\text {out }}, \psi_{T}$, and $\psi_{a v}$ resulting from $\psi_{\text {in }}(x, z)$, there are also analogous equations for the angular fluxes resulting from $\psi_{L}(y, z)$ and $\psi_{B}(x, y)$. Summing these individual contributions along with the contribution from $S(x, y, z)$ yields the desired results for $\psi_{R}, \psi_{\text {out }}, \psi_{T}$, and $\psi_{a v}$.

The next step in this development is to find the contribution of $S(x, y, z)$ to $\psi_{\text {out }}$. This is done by considering differential volumes at different values of $y$. The mapping of the surface from which the source leaks without collision is shown in Figure 7.3. The parametric equations for point $A$ in the figure are

$$
\begin{aligned}
& x=\Delta x\left(1-w_{x} u\right), \\
& y=\Delta y\left(1-w_{y} u\right),
\end{aligned}
$$




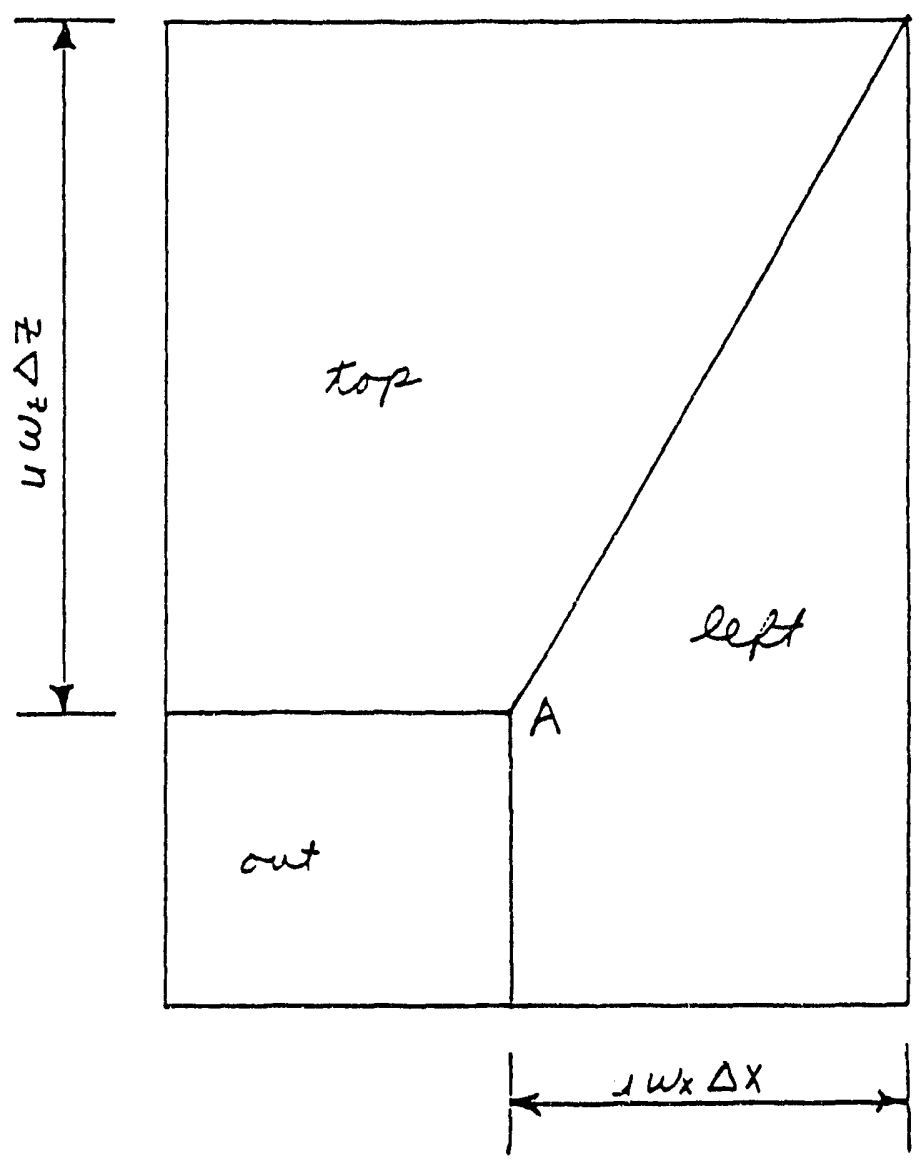

Figure 7.3 Leakage surface by location for sources in $d y$ about $y$. 


$$
\text { and } z=\Delta z\left(1-w_{z} u\right)
$$

The integral expression for $\psi_{\text {out }}$ is

$$
\xi \psi_{\text {out }} \Delta x \Delta z=\int_{\left(1-w_{y}\right) \Delta y}^{\Delta y} e^{-\epsilon u} \int_{0}^{\left(1-w_{x} u\right) \Delta x} \int_{0}^{\left(1-w_{z} u\right) \Delta z} S(x, y, z) d z d x d y
$$

Here $u$ can be considered $u(y)$, where the relationship between $u$ and $y$ is given by Equation (7.23b). The expression to be used for $S(x, y, z)$ is

$$
\begin{gathered}
S(x, y, z)=S_{a v}\left[\left(1-\tilde{S}^{x}\right)+\tilde{S}_{x} \frac{2 x}{\Delta x}\right]\left[\left(1-\tilde{S}^{y}\right)+\tilde{S}_{y} \frac{2 y}{\Delta y}\right]\left[\left(1-\tilde{S}^{z}\right)+\tilde{S}_{z} \frac{2 z}{\Delta z}\right] \\
\text { where } \quad \tilde{S}^{x} \equiv \frac{S^{x}}{S_{a v}} \\
\tilde{S}^{y} \equiv \frac{S^{y}}{S_{a v}} \\
\text { and } \tilde{S}^{z} \equiv \frac{S^{z}}{S_{a v}}
\end{gathered}
$$

The " " will be dropped in the following development since both $S^{x}$ and $\tilde{S}^{x}$ do not appear together.

Substituting Equation (7.25) into Equation (7.24) and performing the $x$ and $z$ integrals yields

$$
\begin{aligned}
& \psi_{\text {out }}=S_{a v} \frac{1}{\xi} \int_{\left(1-w_{y}\right) \Delta y}^{\Delta y} e^{-\epsilon u}\left[\left(1-S^{y}\right)+S^{y} \frac{2 y}{\Delta y}\right] \\
& \left(1-w_{x} u\right)\left(1-w_{x} S^{x} u\right)\left(1-w_{z} u\right)\left(1-w_{z} S^{z} u\right) d y
\end{aligned}
$$

A change of variable from $y$ to $u$ gives

$$
\psi_{\mathrm{out}}=\frac{S_{a v}}{A_{u}} \int_{0}^{1} e^{-\epsilon u}\left[\left(1+S^{y}\right)-2 w_{y} S^{y} u\right]\left(1-w_{x} u\right)\left(1-w_{x} S^{x} u\right)\left(1-w_{z} u\right)\left(1-w_{z} S^{z} u\right) d u
$$


The solution to this equation is

$$
\begin{aligned}
\psi_{\mathrm{out}}=\frac{S_{a v}}{A_{u}}\left\{\left(1-S^{y}\right)\left[\left(H_{3}^{y}-w_{x} S^{x} H_{4}^{y}\right)-w_{z} S^{z}\left(H_{4}^{y}-w_{x} S^{x} H_{5}^{y}\right)\right]\right. \\
\left.+2 S^{y}\left[\left(h_{4}-w_{x} S^{x} h_{5}\right)-w_{z} S^{z}\left(h_{5}-w_{x} S^{x} h_{6}\right)\right]\right\}
\end{aligned}
$$

where $H_{n}^{y}$ is defined in Equation (7.22) and $h_{n}$ is given by

$$
\begin{aligned}
h_{n} & \equiv \int_{0}^{1} u^{n-4} e^{-\epsilon u}\left(1-w_{x} u\right)\left(1-w_{y} u\right)\left(1-w_{z} u\right) d u, n>3 \\
& =F_{n-3}-F_{n-2}\left(w_{x}+w_{y}+w_{z}\right)+F_{n-1}\left(w_{x} w_{y}+w_{x} w_{z}+w_{y} w_{z}\right)-F_{n} w_{x} w_{y} w_{z} .
\end{aligned}
$$

The analogous equations for $\psi_{R}$ and $\psi_{T}$ are

$$
\begin{gathered}
\psi_{R}=\frac{S_{a v}}{A_{u}}\left\{\left(1-S^{x}\right)\left[\left(H_{3}^{x}-w_{y} S^{y} H_{4}^{x}\right)-w_{z} S^{z}\left(H_{4}^{x}-w_{y} S^{y} H_{5}^{x}\right)\right]\right. \\
+2 S^{x}\left[\left(h_{4}-w_{y} S^{y} h_{5}\right)-w_{z} S^{z}\left(h_{5}-w_{y} S^{y} h_{6}\right)\right\}
\end{gathered}
$$

and

$$
\begin{gathered}
\psi_{T}=\frac{S_{a v}}{A_{u}}\left\{\left(1-S^{z}\right)\left[\left(H_{3}^{z}-w_{y} S^{y} H_{4}^{z}\right)-w_{x} S^{x}\left(H_{4}^{z}-w_{y} S^{y} H_{5}^{z}\right)\right]\right. \\
\left.+2 S^{z}\left[\left(h_{4}-w_{y} S^{y} h_{5}\right)-w_{x} S^{x}\left(h_{5}-w_{y} S^{y} h_{6}\right)\right]\right\}
\end{gathered}
$$

where $H_{n}^{x}=F_{n-2}-F_{n-1}\left(w_{y}+w_{z}\right)+F_{n} w_{y} w_{z}$

$$
\text { and } H_{n}^{z}=F_{n-2}-F_{n-1}\left(w_{x}+w_{y}\right)+F_{n} w_{x} w_{y} \text {. }
$$

The applicable balance equation is a special case of Equation (7.16):

$$
\epsilon \psi_{a v}=\frac{S_{a v}}{A_{u}}-\left(w_{x} \psi_{R}+w_{y} \psi_{\mathrm{out}}+w_{z} \psi_{T}\right)
$$


Substituting Equation (7.28), (7.30), and (7.31) into Equation (7.32) and using Equation (7.20) and very lengthy algebra yields

$$
\begin{aligned}
\psi_{a v}= & \frac{S_{a v}}{A_{u}}\left\{\left[\left(h_{4}-w_{x} S^{x} h_{5}\right)-w_{y} S^{y}\left(h_{5}-w_{x} S^{x} h_{6}\right)\right]\right. \\
& \left.-w_{z} S^{z}\left[\left(h_{5}-w_{x} S^{x} h_{6}\right)-w_{y} S^{y}\left(h_{6}-w_{x} S^{x} h_{7}\right)\right]\right\}
\end{aligned}
$$

The equations developed in this section and other analogous ones give the contributions to $\psi_{a v}, \psi_{R}, \psi_{\text {out }}$, and $\psi_{T}$ from $S(x, y, z), \psi_{L}(y, z), \psi_{\text {in }}(x, z)$, and $\psi_{B}(x, y)$. Summing these individual contributions gives the desired values for these four average quantities. However, there are nine additional unknowns $\psi^{x}, \psi^{y}, \psi^{z}, \psi_{R}^{y}, \psi_{R}^{z}, \psi_{\text {out }}^{x}, \psi_{\text {out }}^{z}, \psi_{T}^{x}, \psi_{T}^{y}$ and only three equations, the $x, y$, and $z$ moment balance equations (Equations (2.5)). The current implementation in TORT uses the linear nodal equations given by Equations (3.2) and (2.14) and their analogs to obtain values for the nine unknowns.

Another way to obtain $\psi^{x}$ is from Equation (2.10), and $\psi^{y}$ and $\psi^{z}$ can be obtained similarly. This would leave six unknowns and three equations, so three more supplimental equations would be needed. Characteristic solutions can also be considered for the nine unknowns but at the cost of increased complexity and longer computing times. Further research on this topic could result in additional gains in speed and/or accuracy.

The final development needed for implementing the linear characteristic method is a way to evaluate $F_{n}$. The approach used in TORT is to introduce the functions

$$
f_{n} \equiv e^{\epsilon} F_{n} .
$$

Multiplying Equation (7.19) by $e^{\epsilon}$ yields

$$
f_{n+1}=\frac{n f_{n}-1}{\epsilon} .
$$

Another form of this equation is

$$
f_{n}=\frac{\epsilon f_{n+1}+1}{n} .
$$

In TORT, the approximation $f_{9}=0$ is made, and Equation (7.36) is used to evaluate $f_{8}, f_{7}, \cdots, f_{1}$. 
Equation ( 18$)$ is multiplied, by $e^{\epsilon}$ and rearranged to obtain

$$
e^{\epsilon}=\epsilon f_{1}+1 \text {. }
$$

The approximation for $e^{-\epsilon}$ obtained by this method is

$$
e^{-\epsilon}=\frac{1}{1+\epsilon+\frac{\epsilon^{2}}{2}+\frac{\epsilon^{3}}{3 !}+\frac{\epsilon^{4}}{4 !}+\frac{\epsilon^{5}}{5 !}+\frac{\epsilon^{6}}{6 !}+\frac{\epsilon^{7}}{7 !}+\frac{\epsilon^{8}}{8 !}} .
$$

This approximation is always positive and quite accurate for transport calculation purposes.

Additional identities that are useful for efficient implementation and in derivations are

$$
\begin{aligned}
& H_{n}^{x}=G_{n-1}^{z}-w_{y} G_{n}^{z}=G_{n-1}^{y}-w_{z} G_{n}^{y}, \\
& H_{n}^{y}=G_{n-1}^{x}-w_{z} G_{n}^{x}=G_{n-1}^{z}-w_{x} G_{n}^{z}, \\
& H_{n}^{z}=G_{n-1}^{x}-w_{y} G_{n}^{x}=G_{n-1}^{y}-w_{x} G_{n}^{y},
\end{aligned}
$$

and

$$
h_{n}=H_{n-1}^{x}-w_{x} H_{n}^{x}=H_{n-1}^{y}-w_{y} H_{n}^{y}=H_{n-1}^{z}-w_{z} H_{n}^{z} .
$$




\section{TESTING AND APPLICATIONS}

In the 1984 report of nodal testing in DOT [5], a difficult sodium, steel, concrete, and void problem taken from problem 6 of a 1982 Pevey report [12] was solved. This problem was, roughly, an $\mathrm{XZ}$ geometry version of the DOT test problem 8 . The void region opens directly to the outer boundary, testing the robustness of a given method in the face of severe flux distortion. Table 8.1 shows the results of a meshrefinement study comparing nodal with weighted difference. Solution time, top leakage, and estimated error are shown for both methods using eight mesh sizes. In each case, nodal provides a much more accurate result at somewhat higher solution cost. The report notes that, for $10 \%$ accuracy, nodal uses $1 / 3$ of the time that weighted difference uses, and for $1 \%$ accuracy, nodal uses only $1 / 10$ as much time.

The nodal option is no longer available in our 2-d codes, since $\mathrm{XZ}$ geometry meets only limited use. It could be modernized and restored if a need for it were found, however. The numerical results would be different, of course, due to improvements in the method as discussed below.

In the 1985 report of nodal testing in TORT [6], a fictitious conr te building was subjected to flux streaming through the windows and around obstacles inside. Anomalous flux distortions shown in Figures 4.2 and 4.3 lead to the implementation of the step limit and to 3-D positivity constraints corresponding to those used in 2-D. With these improvements, the nodal method gave stable and reliable results.

In the 1985 paper, results were also presented for a small XYZ model of a building actually constructed and tested at ORNL in 1984 during the TORT Validation Experiment (TVE) [13]. The TVE building was constructed in front of the Tower Shield Reactor at Oak Ridge, and flux from the reactor was allowed to stream in through an open window and around a support pillar inside. A plan view is shown in Figure 8.1. As shown in Figure 8.2, four methods were used to calculate flux at the rear of the building (at the wall opposite the window) as a function of mesh size. This figure illustrates the usual poor performance of the 0 -weighted method for large mesh spacing. The theta-weighted method is no better for the largest meshes, and it improves rapidly with mesh refinement. The "nodal (positive)" method includes a constraint to completely prevent the production of negative results, while the "nodal (relaxed)" method has a version of that constraint that usually, but not always, prevents negatives. Both nodal methods show marked superiority to the weighted methods, with the relaxed restraint being the more accurate. The figure indicates that all methods except 0 -weighted agree for the finest mesh actually tried. It is plausible that all four methods converge at infinitesimal mesh size, as indicated by the dashed extrapolation, but that is not proven, of course. 


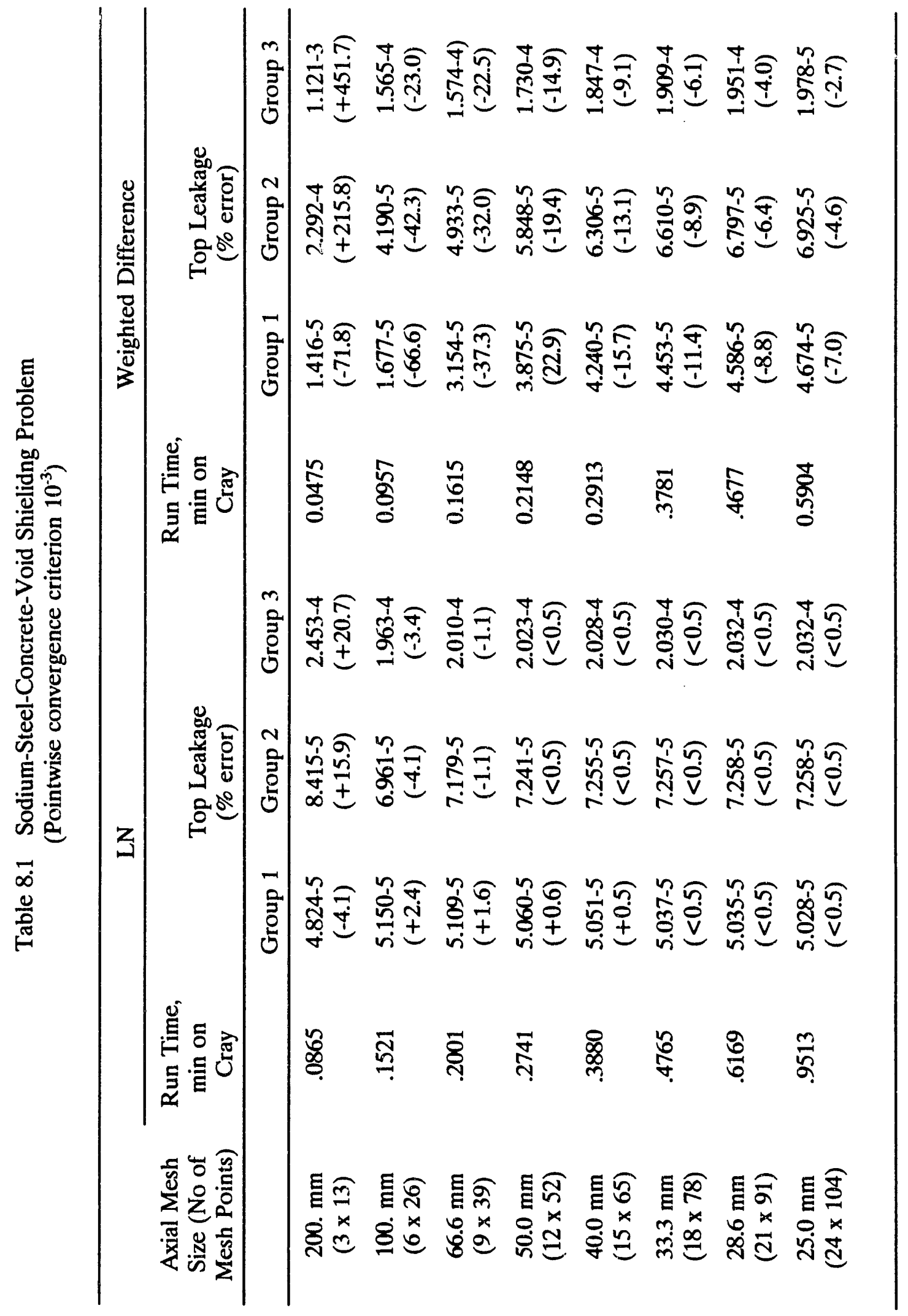




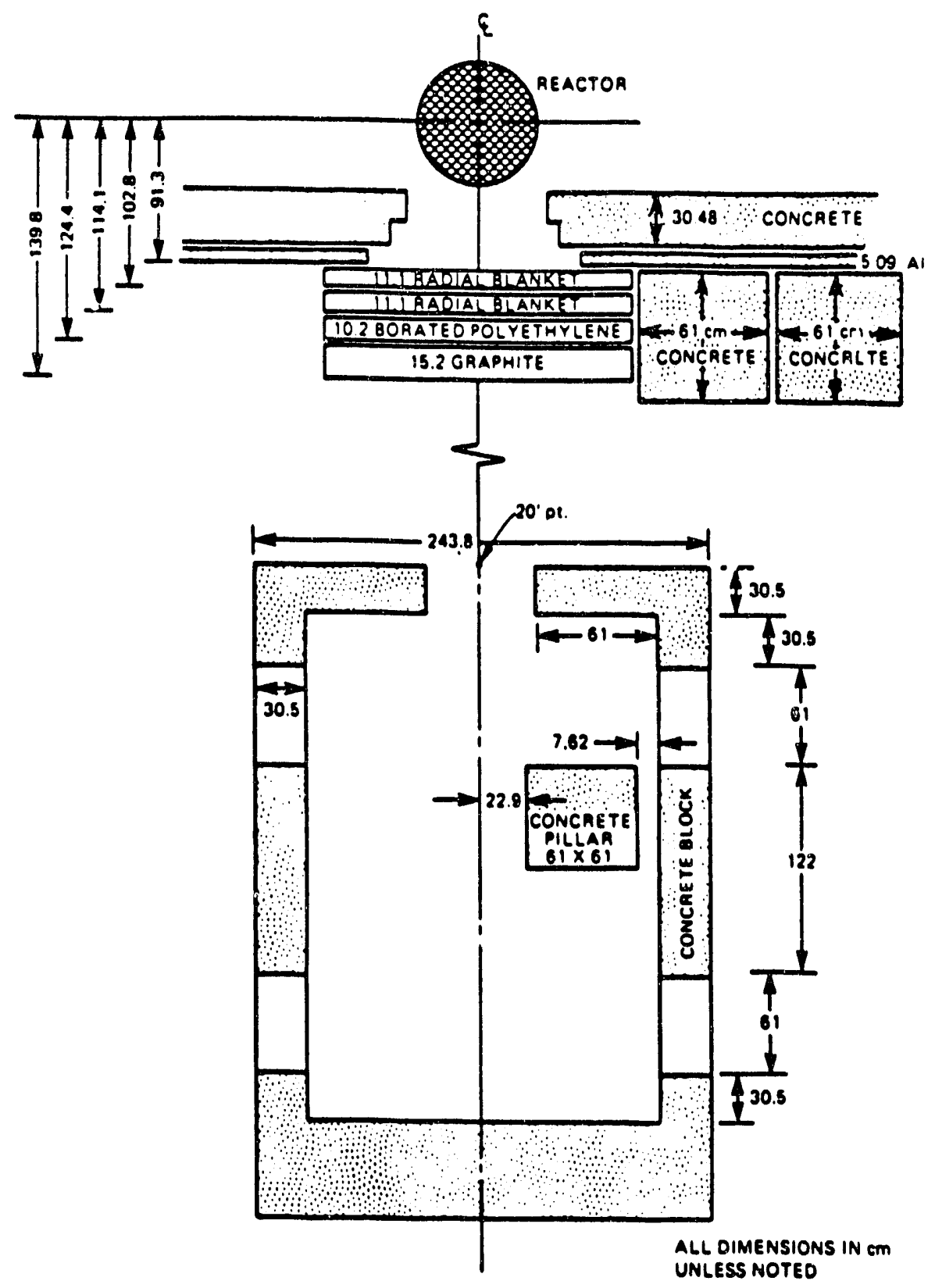

Figure 8.1 Plan View of TVE Concrete Building. 


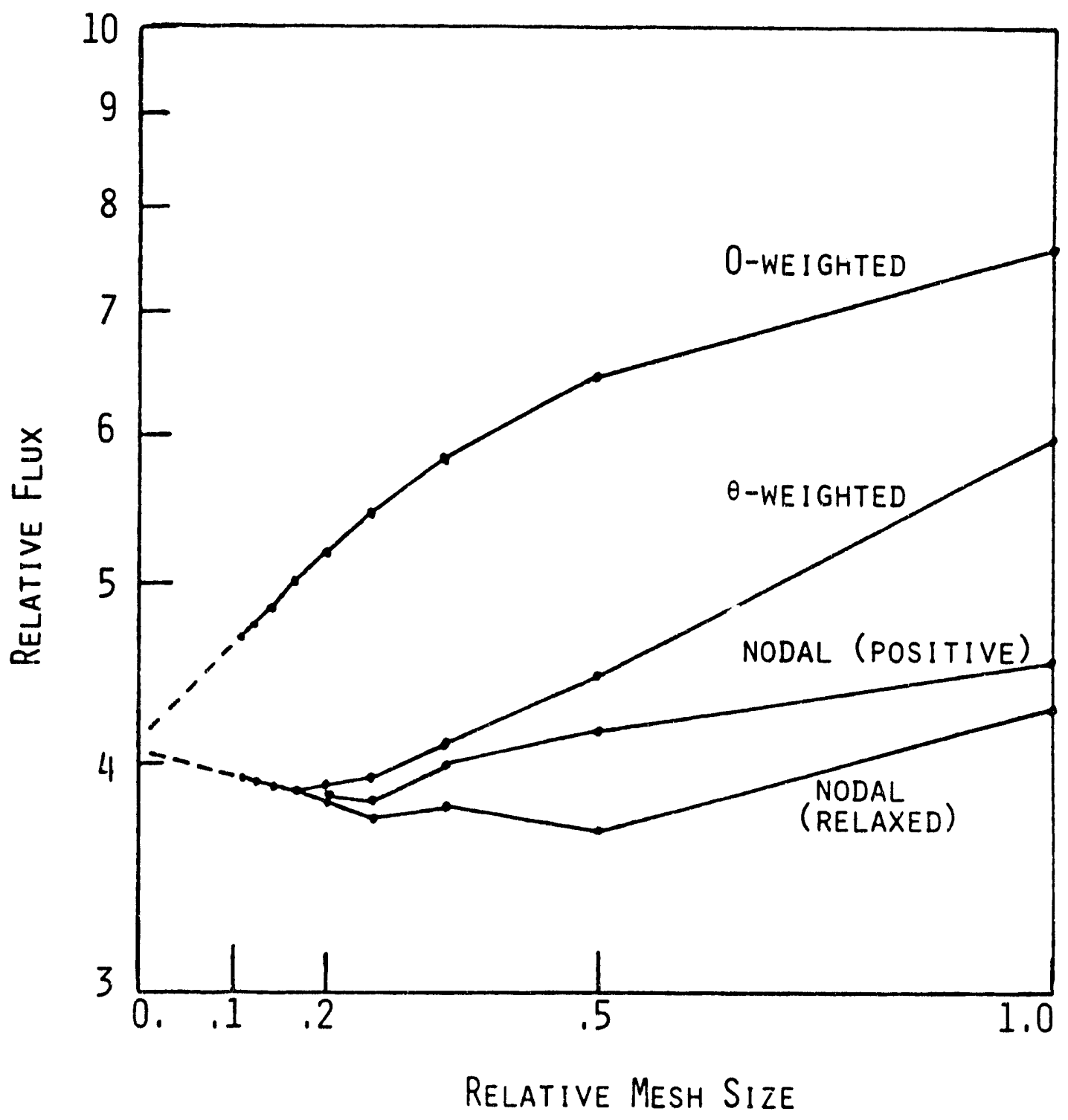

Figure 8.2 Flux In Zone Located In The Back, Center Above The Window Versus Mesh Size. 
In a 1987 paper [9] the TVE building was studied once again, this time with the addition of a linear characteristic method. In this study, maximum error at any air location in the building was studied, rather than error at a specific point. The results, shown in Figure 8.3, show the important advantage of nodal over weighted difference at all mesh sizes once again. Also, the relaxed nodal method was again superior to the positive nodal in the problem studied. The linear characteristic method, also a positive method, is seen to be far superior to the others in this case.

It may be useful at this point to relate the TORT options discussed with the derivations of the previous chapters. The fully-positive and relaxed-constraint nodal procedures are accomplished in the standard nodal subroutines by adjusting a parameter between 0 and 1 . This parameter was originally input using the same name, THETA, as the adjustment parameter needed for the theta-weighted procedure. As the circle of users grew, this lead to misunderstanding, and the nodal adjustment was moved to a new variable, WNODAL, in version 2.2 .10 . In either case, a value of 0 produced the constraint implied by Equation 5.8, and a value of 1 produced the result of 5.9. The constraint is continuously variable between those limits. Both vector and scalar coding use this formulation.

In recent versions, a "new nodal" option has been added. This uses the constraints implied by Equation 5.11. Its results are independent of any adjustable parameter, it does not provide negatives, and preliminary testing shows it to be approximately as accurate as the relaxed nodal method.

Some comparisons have been performed to measure the execution speeds of the procedures. The problem selected was the TORT demonstration problem 6 , a concrete building $70 \mathrm{~m} \times 17 \mathrm{~m} \times 15 \mathrm{~m}$ high. A computer model of this building is shown in Figure 8.4. The mesh required 104,247 cells. One group of a 20-group cross section set was used in a P-3 calculation. The symmetrical S-6 quadrature had 60 directions. The computing times for a Cray Y-MP mainframe computer and an IBM RS/6000 workstation are shown in Table 8.2, and the computing speeds are shown in Table 8.3. It may be noted that the theta-weighted difference method used partial vector coding and a Cray Assembler Language (CAL) subroutine on the Cray. It would have been much slower without that help. Also, the standard nodal method was vectorized on the Cray, but vector versions of the other nodal routines were not available. The characteristic calculations were entirely scalar for the same reason. The "unsafe" nodal used for the table was the standard method with the constraints on boundary slopes removed. It is presented for the sake of comparison with unconstrained methods in other codes.

Table 8.2 indicates the relative value of a key flux at the center of the building on the first floor. At this point, the flux has attenuated almost a factor of 100 from 


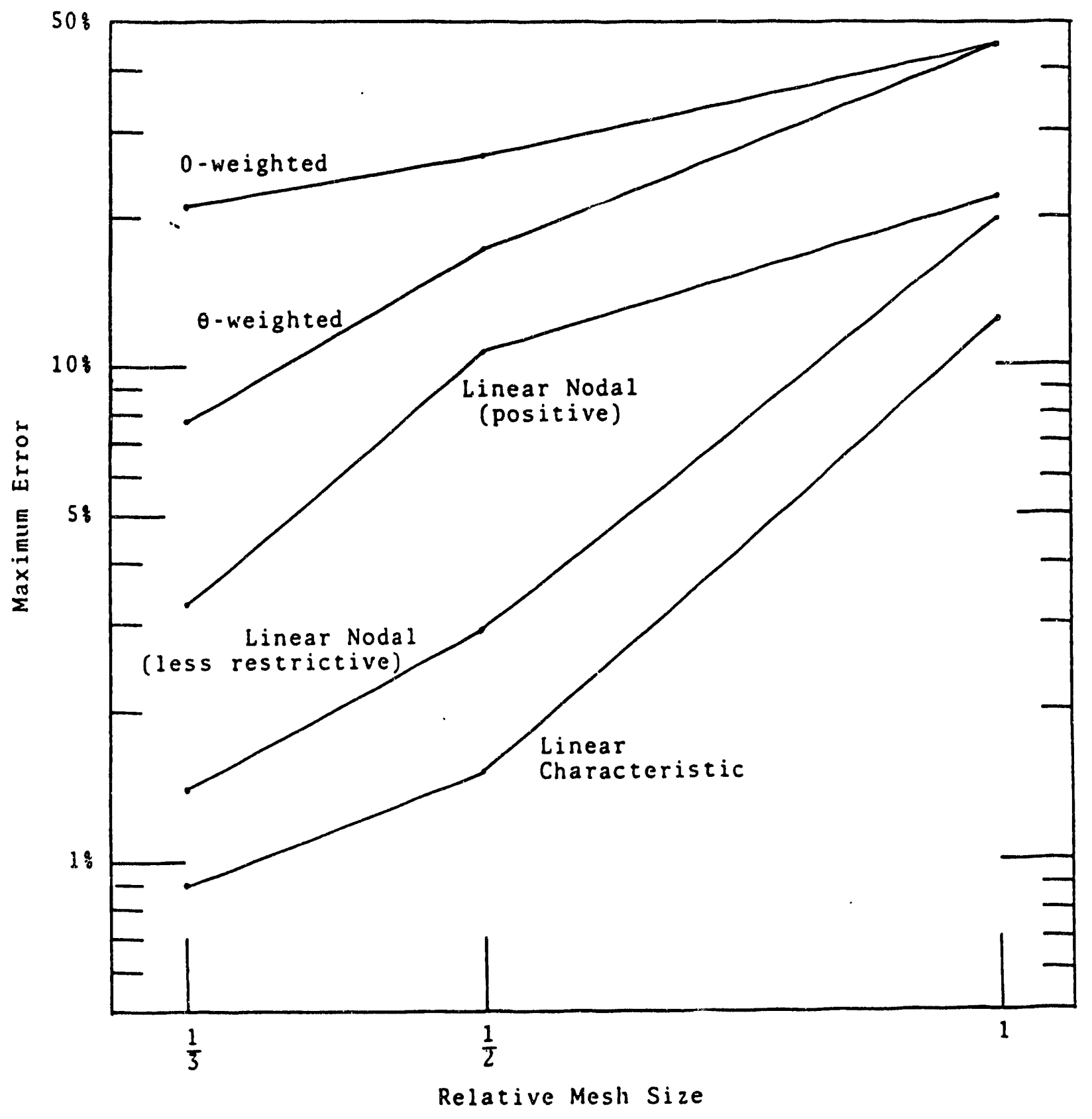

Figure 8.3 Maximum Error for Different Methods and Mesh Size. 


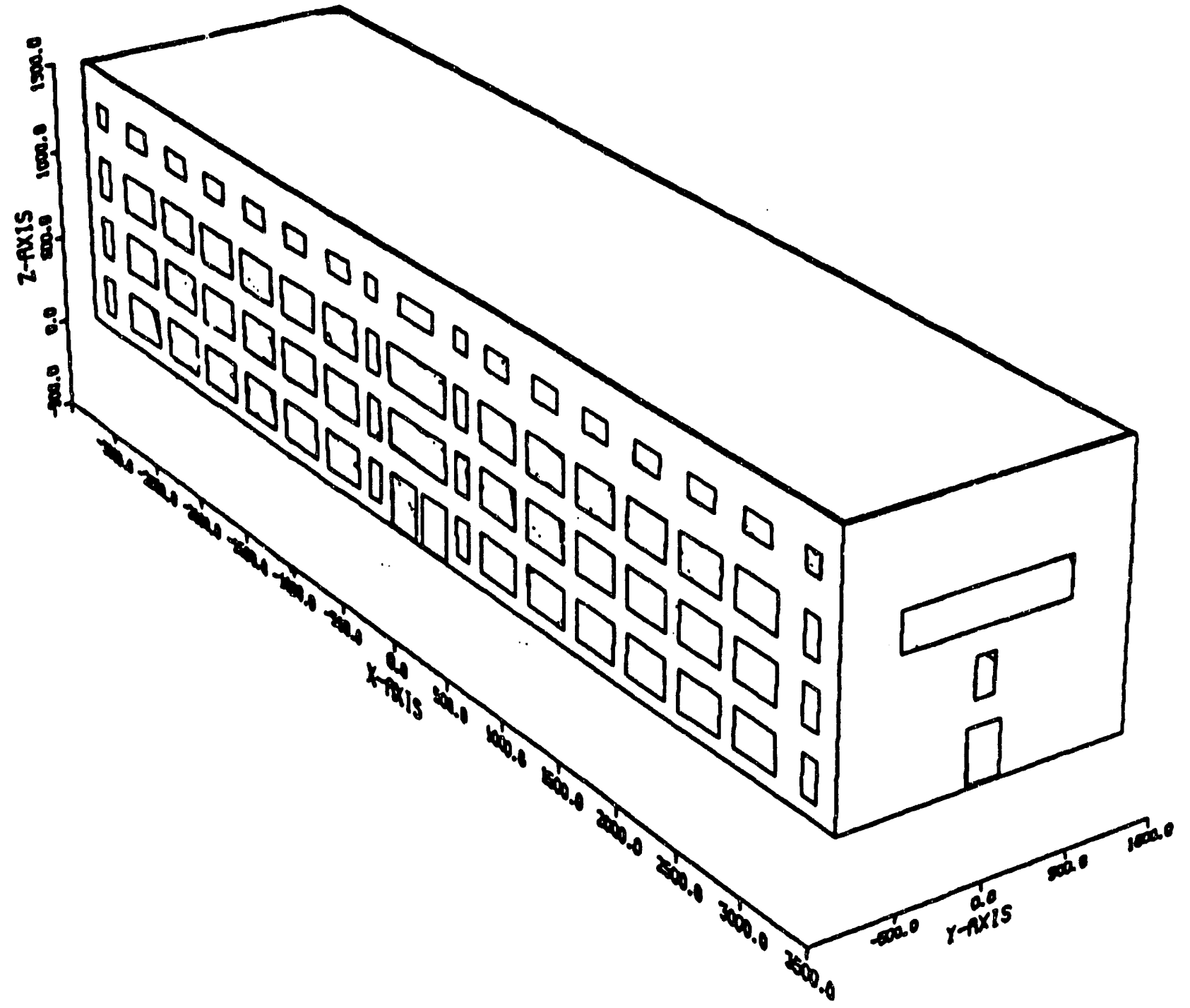

Figure 8.4 Model of a Large Concrete Building. (Dimensions in cm) 
Table 8.2 Timing Results for Flux Procedures

\begin{tabular}{|c|c|c|c|}
\hline & $\begin{array}{c}\text { IBM } \\
\text { microsec/flux }\end{array}$ & $\begin{array}{c}\text { Cray } \\
\text { microsec/flux }\end{array}$ & $\begin{array}{l}\text { Key flux } \\
\text { (relative) }\end{array}$ \\
\hline $\begin{array}{l}\text { Theta-weighted } \\
\text { (vector +cal) }\end{array}$ & $\cdots$ & 1.85 & 2.61 \\
\hline (scalar) & 15.9 & $\cdots$ & 2.61 \\
\hline $\begin{array}{c}\text { Characteristic } \\
\text { (scalar) }\end{array}$ & 52.4 & 21.9 & 2.52 \\
\hline $\begin{array}{l}\text { Nodal (positive) } \\
\text { (vector) }\end{array}$ & & 4.52 & 2.41 \\
\hline (scalar) & 57.1 & $\ldots$ & 2.41 \\
\hline $\begin{array}{l}\text { Nodal (relaxed) } \\
\text { (vector) }\end{array}$ & & 4.52 & 2.35 \\
\hline (scalar) & 57.2 & $\cdots$ & 2.35 \\
\hline $\begin{array}{l}\text { Nodal(new) } \\
\text { (scalar) }\end{array}$ & 56.5 & 14.5 & 2.43 \\
\hline $\begin{array}{l}\text { Nodal (unsafe) } \\
\text { (scalar) }\end{array}$ & 38.6 & 8.64 & 2.15 \\
\hline
\end{tabular}

Notes:

- The precompiler was not used on either machine.

- The IBM results are for an RS/6000 model $320 \mathrm{~h}$. Tests indicate that a model 220 might run $40 \%$ longer, while a model 550 might run roughly half as long.

- The Cray results are for a Y-MP.

- The unsafe nodal did not converge in 15 iterations. 
Table 8.3 Computing Speed for Flux Procedures

\begin{tabular}{||c|c|c|c||}
\hline \multicolumn{2}{|c}{ Flops per flux } & $\begin{array}{c}\text { IBM } \\
\text { Mflops/sec }\end{array}$ & $\begin{array}{c}\text { Cray } \\
\text { Mflops/sec }\end{array}$ \\
\hline $\begin{array}{c}\text { Theta-weighted } \\
\text { (vector+cal) } \\
\text { (scalar) }\end{array}$ & 141 & $\ldots$ & 76 \\
\hline $\begin{array}{c}\text { Characteristic } \\
\text { (scalar) }\end{array}$ & $108-116$ & 6.8 & $\ldots$ \\
\hline $\begin{array}{c}\text { Nodal (positive) } \\
\text { (vector) } \\
\text { (scalar) }\end{array}$ & 426 & 8.1 & 19 \\
\hline $\begin{array}{c}\text { Nodal (relaxed) } \\
\text { (vector) } \\
\text { (scalar) }\end{array}$ & 302 & $\ldots$ & 67 \\
\hline $\begin{array}{c}\text { Nodal(new) } \\
\text { (scalar) }\end{array}$ & 302 & 4.7 & 67 \\
\hline $\begin{array}{c}\text { Nodal (unsafe) } \\
\text { (scalar) }\end{array}$ & 266 & 4.7 & $\ldots$ \\
\hline
\end{tabular}

Notes:

- The theta-weighted procedure used a variable number of flops, depending upon whether negatives are discovered. The smaller value is used here.

- Only 48 directions contributed flops. The other directions had zero weight, and their calculation was bypassed.

- A "flop" is a floating point operation, i.e., add, subtract, multiply, or divide. 
the building surface. All of the methods that converged gave relatively consistent results for this point in spite of the difficulties imposed by large mesh sizes and the alternating pattern of windows and thick concrete. Other results indicate important differences near the pillars between the windows, where the larger numerical dispersion inherent to weighted difference scatters flux into the shadow area. For this and other reasons, the customer for this calculation required the added accuracy of nodal. A discussion of numerical dispersion is given in the TORT document [1]. Production results using somewhat more elaborate building models were reported in an ORNL report and in a conference paper. [14,15]

A KAPL analysis of a streaming experiment of an iron-lined duct embedded in polyethylene showed that the nodal procedure produced good results with only $8 \%$ of the computing time required by weighted difference. [16] One suspects that the improved numerical dispersion properties may be important in this application, as well as the ability to calculate thick mesh spacing accurately.

Reactor analysts have also made good use of the new methods. In a study of neutron exposure in the HFIR reactor, the 2-d DORT code was used to calculate flux at a surface surrounding experimental penetrations (Figure 8.5), and then TORT nodal calculations were used to calculate the streaming through the void tubes and into the vessel adjacent to the tubes. $[17,18]$ The objective was to relate experimental data in test samples near the vessel, as indicated in the figure, to flux in the vessel steel, where experiments were not possible. The calculation also provided essential spectral information that could not be observed experimentally. The calculated results are compared with the observations in Table 8.4. It was necessary to exclude three of the samples due to inconsistent nickel-to-iron ratios, a certain indication of experimental error. The remaining calculations fit the observations with a normalization of 0.69 and a fractional standard deviation of $10 \%$. This was considered an excellent fit, considering that the samples differed by a factor of 5 in their exposure, and it was sufficient to accomplish the objectives. The normalization apparently resulted from the DORT core calculation, not from the nodal TORT analysis.

The importance of ray effects in this calculation was examined in some detail. It was found that a biased quadrature set was required to calculate a proper flux shape in the void tube, although that treatment was not used in the calculations reported here.

The characteristic calculations have not been used extensively on large-scale production applications. They are newer, and potential users have not built a feeling of confidence in them, as compared to the nodal procedures. Also, successful vector routines for the characteristic procedure have not been constructed, although this is 


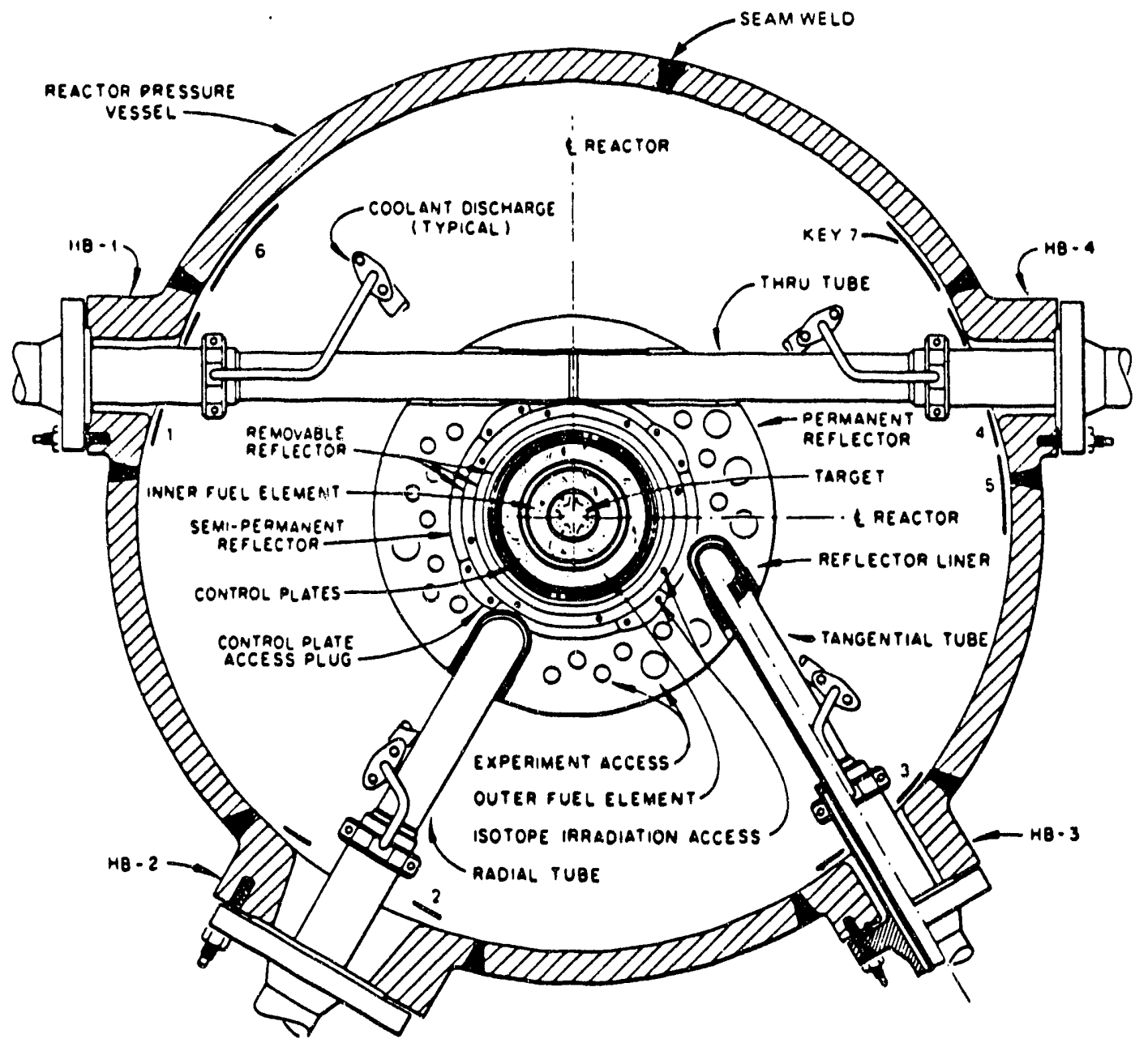

Figure 8.5 Cross Section of HFIR Vessel Showing Locations of Surveillance Capsules. 


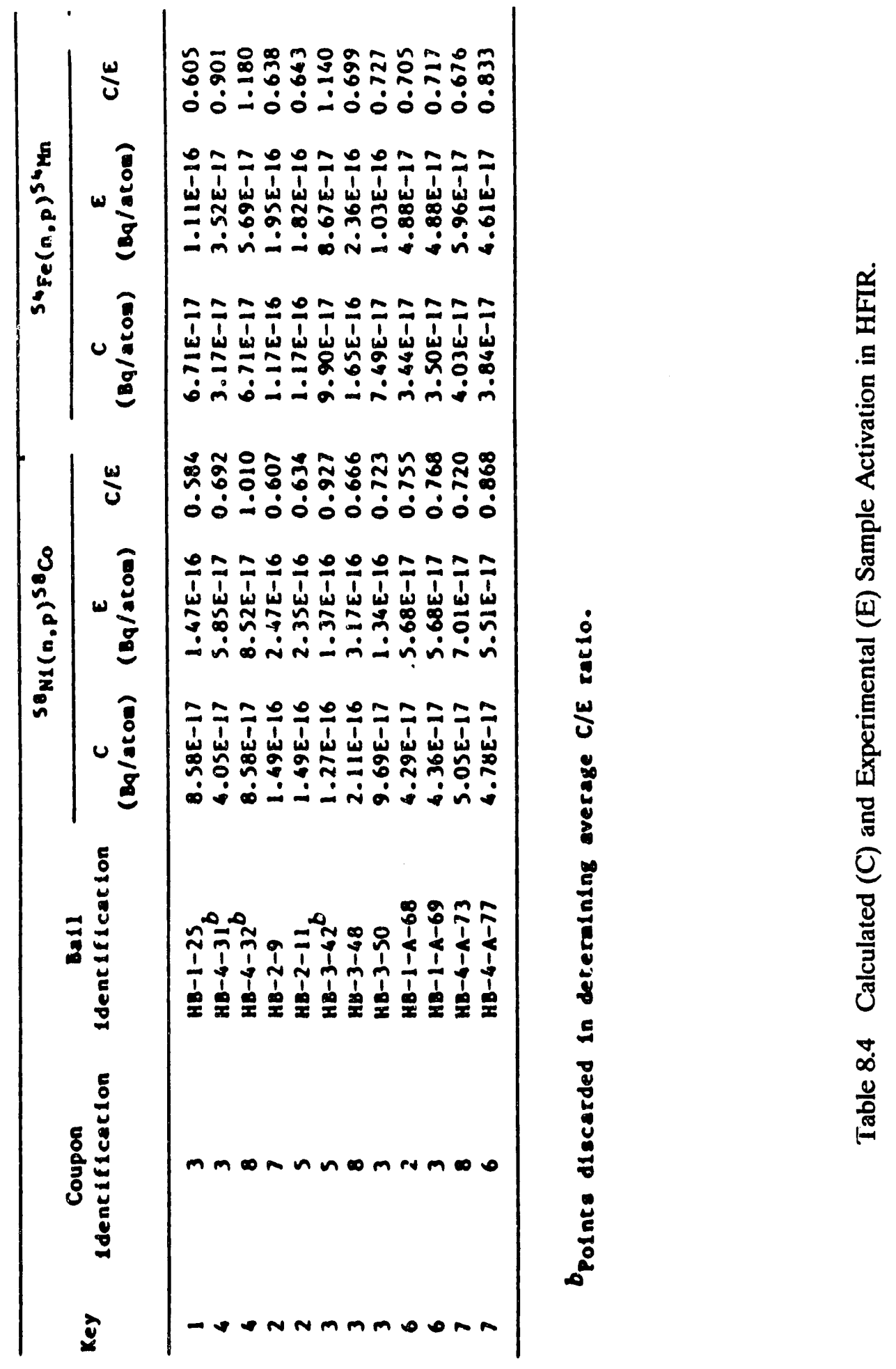


potentially feasible. Our existing characteristic routine is actually faster than nodal on the IBM scalar work stations, however, and that should encourage increased application in the future.

It may be noted that the increased work required to perform nodal or characteristic procedures make them good candidates for parallel computers. A 1989 paper found that adjacent directions could be calculated in parallel, resulting in more efficient use of computer resources. [19] Although the actual coding that accomplished the parallel testing is obsolete, it could be modernized in a new computer environment as required.

Finally, we note abstracts of newer versions of TORT published in 1991 [20,21]. Both versions contained nodal and characteristic routines, but the latter version was adapted to IBM workstations as well as Cray computers. Compatibility with SUN and HP workstations has been demonstrated since that time. 


\section{CONCLUDING COMMENTS}

The basic idea involved in nodal and characteristic methods is that an analytical solution is performed over a relatively large mesh cell containing only one type of material, and then the flux shapes at the boundaries are linked to adjacent cells by using suitable approximations, such as polynomial expansions. This also requires that the source within the cell be approximated similarly. When this is done, the limit on mesh cell size imposed by conventional finite difference methods is overcome, and the limits are only those imposed by material discontinuity or by limitations in the accuracy of the boundary and source approximations.

Applications of this idea date back at least to the 1960's [22], but more-powerful computing machines and refined mathematics were required to make the methods practical. The linear nodal method pioneered by Walters and discussed herein is more than 10 year old, and it has taken its place as a competitor to, or possibly a replacement for, the weighted difference method that has been the mainstay of radiation transport since the mid-1970's.

Initial testing of the basic linear nodal method on difficult problems typical of radiation transport uncovered limitations that would limit its acceptance. The addition of the positivity constraints and the step limit controlled these difficulties, however, and nodal calculations have proven at least as robust as weighted difference. In addition, they have proven to be much more tolerant of the large mesh sizes common in ex-core transport work.

In some applications, nodal methods are viewed as allowing larger mesh spacing, with a mesh-cubed savings in computer costs in 3-dimensions. In other applications, the mesh size is fixed by geometric or economic constraints, and the nodal approach provides improved accuracy. In still others, the weighted difference method is found adequate, but the value of nodal is in providing independent confirmation of that adequacy.

The characteristic method shows improved accuracy as compared to nodal in many tests. Its applications have been slower to grow, in part because it is less familiar, and in part because a vectorized version is not available. The method is actually faster on the IBM RS/6000 workstation, however, and its popularity may grow in the future.

Both methods produce positive results, given positive inputs. They may react badly to negative inputs, however, and this matter must be dealt with in the computer implementation. Acceleration is also a problem, in that the complexity is 
an obstacle to the use of diffusion synthetic acceleration. In the TORT code, the partial current method used with weighted difference has been used successfully in problems that do not depart too far from typical mesh spacing and scattering ratios. The development of a globally-effective acceleration scheme would be important in extending the range of application, however. 


\section{REFERENCES}

1. W. A. Rhoades and R. L. Childs, "The TORT Three-Dimensional Discrete Ordinates Neutron/Photon Transport Code," ORNL-6268 (November 1987).

2. W. F. Walters and R. D. O'Dell, "A Comparison of Linear Nodal, Linear Discontinuous, and Diamond Schemes for Solving the Transport Equation in $(x, y)$ Geometry," Trans. Am. Nucl. Soc. 39, 465-467 (San Francisco, November 1981).

3. W. F. Walters and R. D. O'Dell, "Nodal Methods for Discrete-Ordinates Transport Problems in $(x, y)$ Geometry," Proc. Amer. Nucl. Soc. Top. Meeting on Advances in Mathematical Methods for the Solution of Engineering Problems, Vol. I, 115-129 (Munich, April 1981).

4. W. A. Rhoades and R. L. Childs, "An Updated Version of the DOT 4 One- and Two-Dimensional Neutron/Photon Transport Code," ORNL-5851 (July 1982).

5. R. L. Childs and W. A. Rhoades, "The Linear Nodal Method For Shieiding Applications," Trans. Am. Nucl. Soc. 46, 657-658 (New Orleans, June 1984).

6. R. L. Childs and W. A. Rhoades, "The Extension of the Linear Nodal Method to Large Concrete Buildings," Trans. Am. Nucl. Soc. 50, 476-477 (San Francisco, November 1985).

7. W. F. Walters, "Augmented Weighted Diamond Form of the Linear Nodal Scheme for Cartesian Coordinate Systems," Proc. Topl. Mtg. Advances in Nuclear Engineering Computational Methods, Vol.2, 452 (Knoxville, April 1985).

8. A. Badruzzaman, "An Efficient Algorithm for Nodal-Transport Solutions in Multidimensional Geometry," Nucl. Sci. Eng. 89, 281 (1985).

9. R. L. Childs and W. A. Rhoades, "The Linear Characteristic Method in XYZ Geometry," Trans. Am. Nucl. Soc. 55, 352-354 (Los Angeles, November 1987).

10. R. E. Alcouffe and E. W. Larsen, "A Review of Characteristic Methods Used to Solve the Linear Transport Equation," Proc. Amer. Nucl. Soc. Top. Meeting on Advances in Mathematical Methods for the Solution of Engineering Problems, Vol. I, 3-16 (Munich, April 1981).

11. E. W. Larsen and R. E. Alcouffe, "The Linear Characteristic Method for Spatially Discretizing the Discrete-Ordinate Equations in x,y-Geometry," Proc. Amer. Nucl. Soc. Top. Meeting on Advances in Mathematical Methods for the Solution of Engineering Problems, Vol. I, 99-113 (Munich, April 1981).

12. R. E. Pevey, "Development of a New Two-Dimensional Cartesian Geometry Nodal Multigroup Discrete Ordinates Method," ORNL/CSD/TM-182 (July 1982). 
13. F. J. Muckenthaler, L. B. Holland, J. L. Hull, and J. J. Manning, "Verification Experiment of the Three-Dimensional Oak Ridge Transport Code (TORT)," ORNL/TM-9528 (December 1985).

14. W. A. Rhoades, R. L. Childs, and D. T. Ingersoll, "Radiation Exposure Inside Reinforced Concrete Buildings at Nagasaki," Oak Ridge National Laboratory, Oak Ridge, TN, ORNL/TM-10999 (December 1988). (A shorter version has been prepared for publication in Health Physics 63(5) 510-521 (November 1992).

15. W. A. Rhoades, R. L. Childs, and D. T. Ingersoll, "New Dose-Mortality Data Based on 3-D Radiation Shielding Calculations for Concrete Buildings at Nagasaki," Proc. 7th Internat. Conf. on Radiation Shielding, Bournemouth, UK, (September 1988).

16. D. A. Barnett, Jr., "Benchmark Measurements and Calculations of a ThreeDimensional Neutron Streaming Experiment," KAPL-4721 (February 1991).

17. R. L. Childs, F. B. K. Kam, R. E. Maerker, W. A. Rhoades, L. R. Williams, and C. A. Baldwin, "Neutron Dosimetry Analysis," Appendix E in "Evaluation of HFIR Pressure-Vessel Integrity Considering Radiation Embrittlement," R. D. Cheverton, J. G. Merkle, and R. K. Nanstad, Editors, ORNL/TM-10444 (April 1988).

18. R. L. Childs, W. A. Rhoades, and L. R. Williams, "Three-Dimensional Calculations of Neutron Streaming in the Beam Tubes of the ORNL HFIR Reactor," Proc. 7th Internat. Conf. on Radiation Shielding, Bournemouth, UK, (September 1988)

19. W. A. Rhoades and R. E. Flanery, "3-D Discrete Ordinates Calculatons with Parallel-Vector Processors," Proc. Am. Nucl. Soc. Topical Meeting on Advances in Nuclear Engineering Computation and Radiation Shielding, pp. 69:1-69:11, Santa Fe (April 9-13, 1989).

20. W. A. Rhoades and R. L. Childs, "TORT: A Three-Dimensional Discrete Ordinates Neutron/Photon Transport Code," Nucl. Sci. \& Engr. 107, 4, pp. 397- 398 (April 1991).

21. W. A. Rhoades and R. L. Childs, "TORT - Three-Dimensional Discrete Ordinates Neutron/Photon Transport Code," Proceedings of the Am. Nucl. Soc. Topical Mtg.: Advances in Mathematics, Computations, and Reactor Physics, Pittsburgh, Pa. (April 28 - May 2, 1991).

22. K. D. Lathrop, "Spatial Differencing of the Transport Equation: Positivity vs. Accuracy," Journal of Computational Physics 4, 475-498 (1969). 
ORNL/TM-12246

\section{INTERNAL DISTRIBUTION}

1. Y. Y. Azmy

2. J. A. Bucholz

3-7. R. L. Childs

8-12. W. W. Engle, Jr.

13. D. T. Ingersoll

14. J. V. Pace III

15. C. V. Parks

16. R. T. Primm III

17-22. W. A. Rhoades

23-27. R. T. Santoro

28. C. O. Slater
29. R. M. Westfall

30. EPMD Reports Office

31-32. Laboratory Records

Department

33. Laboratory Records, ORNL-RC

34. Document Reference Section

35. Central Research Library

36. ORNL Patent Section

\section{EXTERNAL DISTRIBUTION}

37. Prof. Roger W. Brockett, Harvard University, Pierce Hall, 29 Oxford Street, Cambridge, MA 02138

38. Prof. John J. Dorning, Department of Nuclear Engr., Univ. of Va. Reactor Facility, Charlottesville, VA 22901

39. Dr. James E. Leiss, Rt. 2 Box 142C, Broadway, VA 22815

40. Prof. Neville Moray, Dept. of Mechanical and Industrial Engr., University of Illinois, 1206 West Green Street, Urbana, IL 61801

41. Prof. Mary F. Wheeler, Department of Mathematics, Rice University, P. O. Box 1892, Houston, TX 77251

42. I. K. Abu-Shumays, Bettis Atomic Power Laboratory, P. O. Box 79, West Mifflin, PA 15122-0079

43. D. A. Barnett, Knolls Atomic Power Laboratory, P. O. Box 1072, Schenectady, NY 12301

44. R. A. Castelli, Knolls Atomic Power Laboratory, P. O. Box 1072, Schenectady, NY 12301

45. M. L. Grit?ner, 10260 Campus Point Drive, San Diego, CA 92212

46. J. Helm, Columbia University, APNE D pt., 202 SW Mudd, New' York, NY 10027

47. C. G. Yehnert, Bettis Atomic Power Laboratory, P. O. Box 79, West Mifflin, PA 15122-0079

48. W. W. Walters, Los Alamos National Laboratory, P. O. Box 1663, Los Alamos, NM 87544

49. R. W. Young, Defense Nuclear Agency, ATTN: RARP, 6801 Telegraph Road, Alexandria, VA 22310

50. Office of Assistant Manager for Energy Research and Development, Department of Energy, Oak Ridge Operations, P. O. Box 2001, Oak Ridge TN 37831

51-60. Office of Scientific and Technical Information, P. O. Box 62, Oak Ridge, TN 37830 

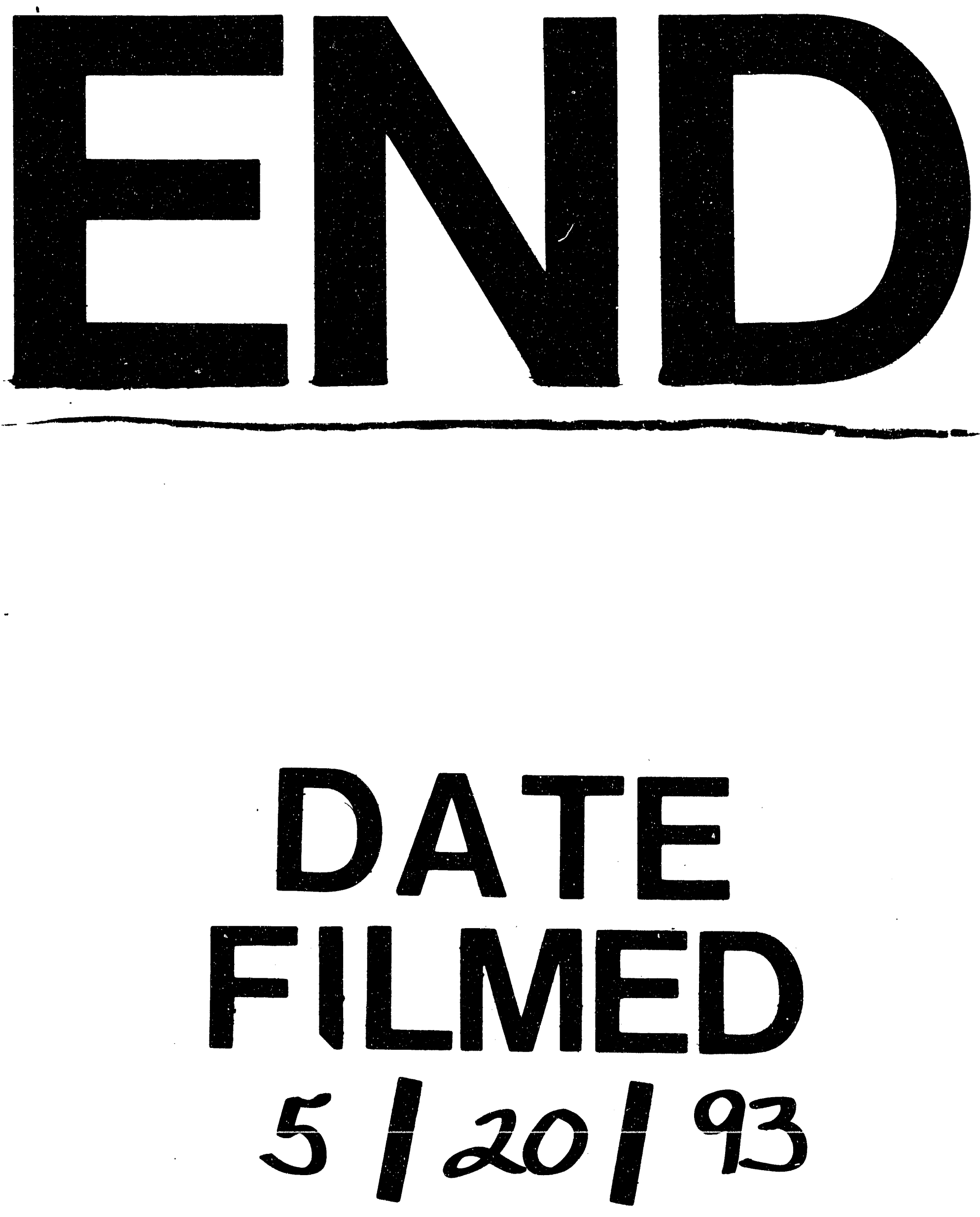
1 